Supplementary Information Submitted to Journal of Physical Chemistry

\title{
A General Strategy to Synthesize Ultrathin Palladium/Transition Metal Alloy Nanowires: Anti-Poisoned Electrocatalytic performance for ORR in acidic and alkaline
}

Hassan Ali, ${ }^{a}$ Haifeng Lin, ${ }^{b}$ Qichen Lu, ${ }^{a}$ Xun Wang $*, a$

${ }^{a}$ Key Lab of Organic Optoelectronics and Molecular Engineering, Department of chemistry, Tsinghua University, Beijing 100084, P. R. China

${ }^{\mathrm{b}}$ Key Laboratory of Eco-Chemical Engineering, Taishan Scholar Advantage and Characteristic Discipline Team of Eco Chemical Process and Technology, College of Chemistry and Molecular Engineering Qingdao University of Science and Technology, Qingdao 266042, P. R. China. 


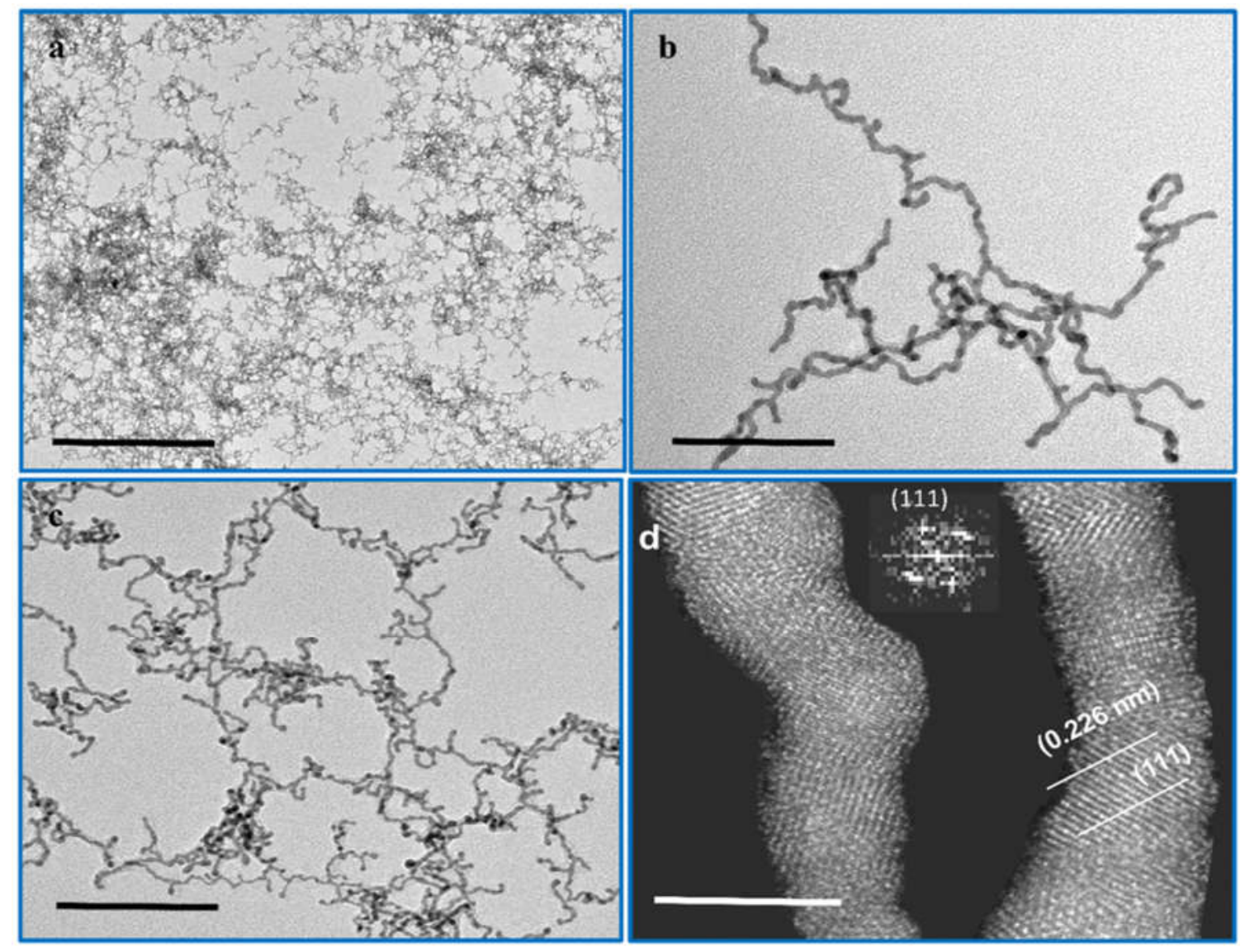

Figure S1. (a-c) TEM images of synthesized $(\mathrm{a}, \mathrm{b}) \mathrm{PdBiCu}$ NWs (scale bar: $1 \mu \mathrm{m}$ and $50 \mathrm{~nm}$ for a and b, respectively) and (c) PdBi NWs (scale bar: $100 \mathrm{~nm}$ ). (d) HAADF-STEM and FFT pattern (inset) of PdBiCu NWs (scale bar: $5 \mathrm{~nm}$ ). 

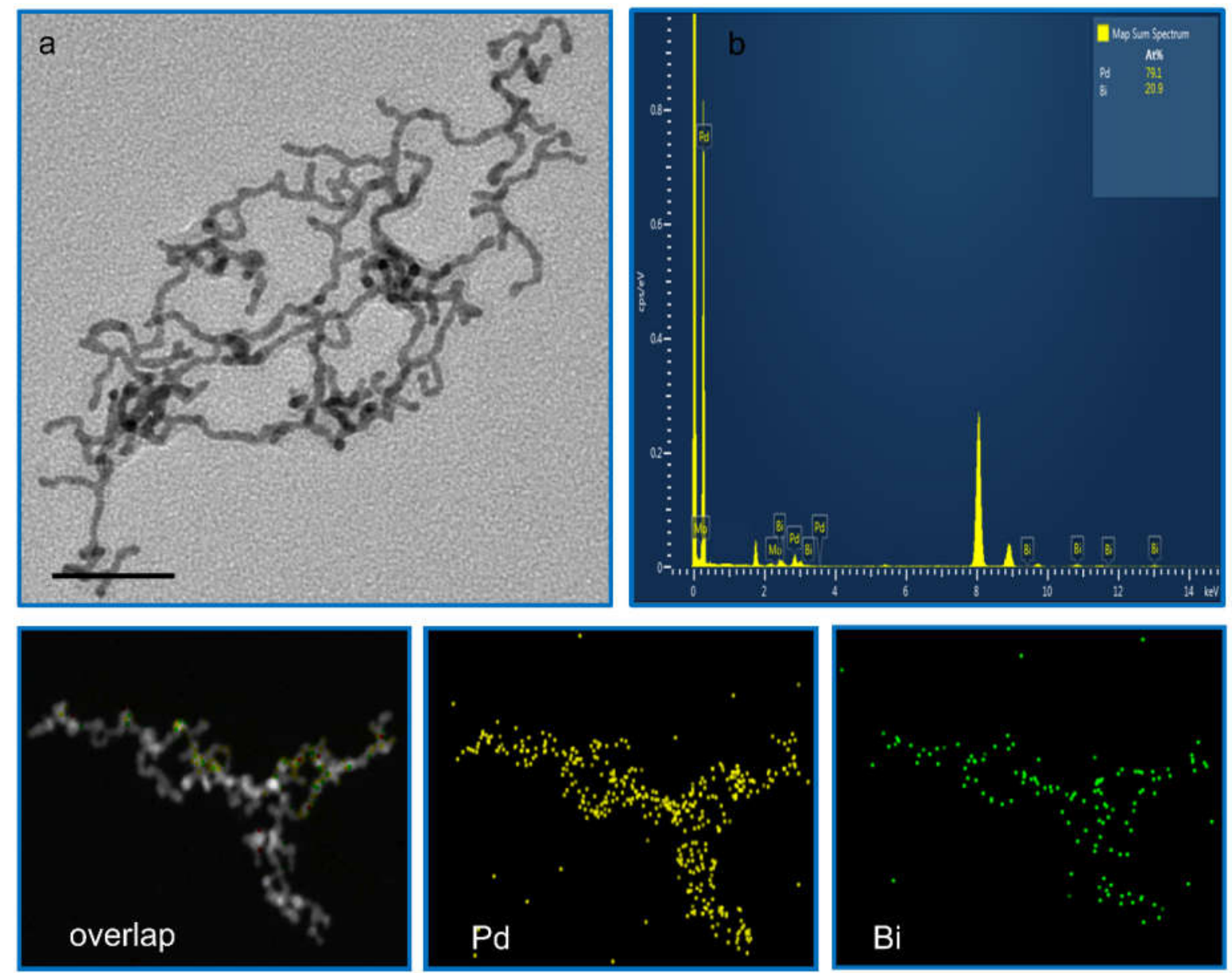

Figure S2. (a) TEM image (scale bar: $50 \mathrm{~nm}$ ) and (b) EDS and elemental mapping results of PdBi NWs. 

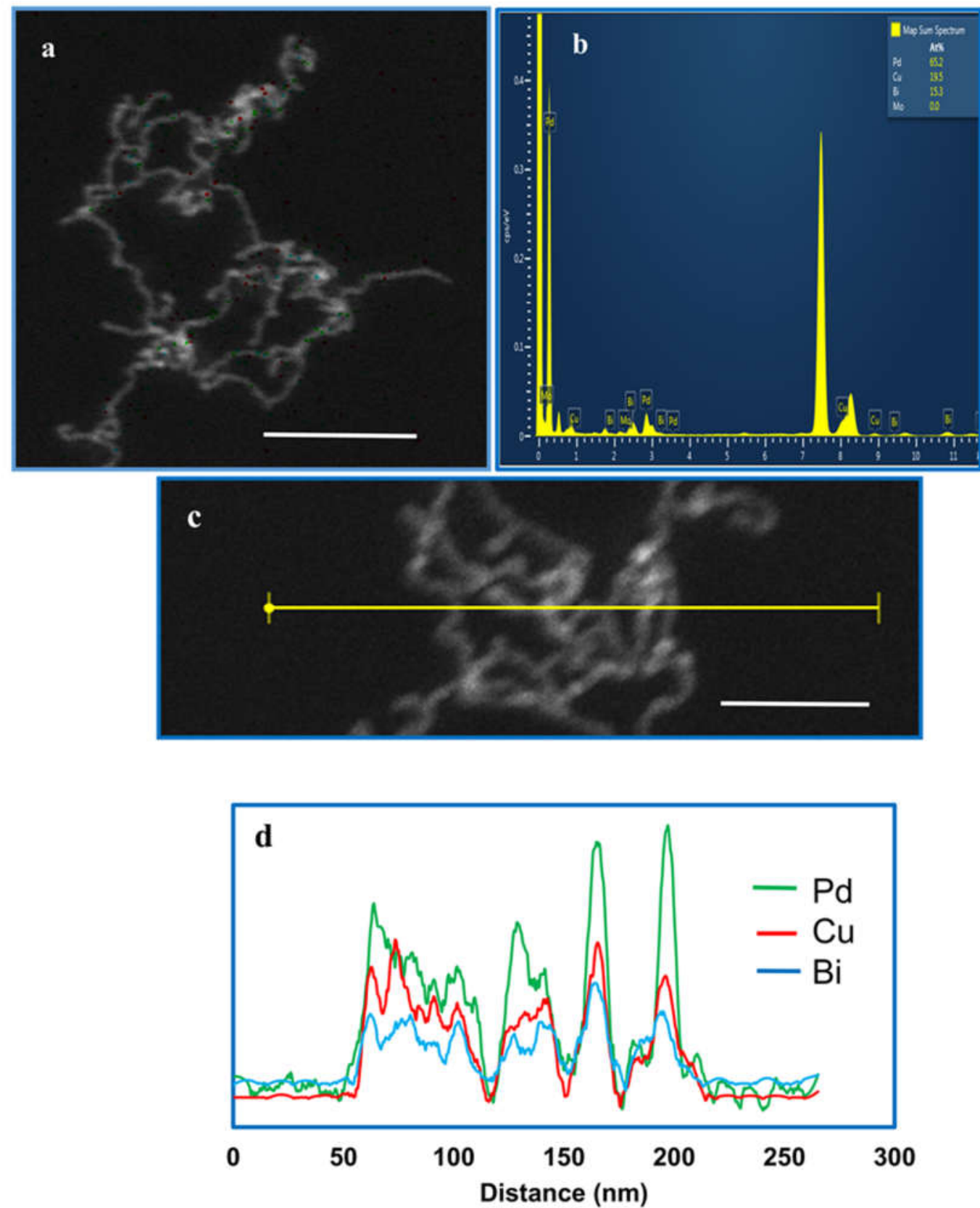

Figure S3. (a) TEM image, (b) EDS spectrum, (c) selected TEM area for line scan and (d) line scanning profile of synthesized ultrathin PdBiCu NWs (scale bar: $100 \mathrm{~nm}$ and $50 \mathrm{~nm}$ for a and c, respectively). 


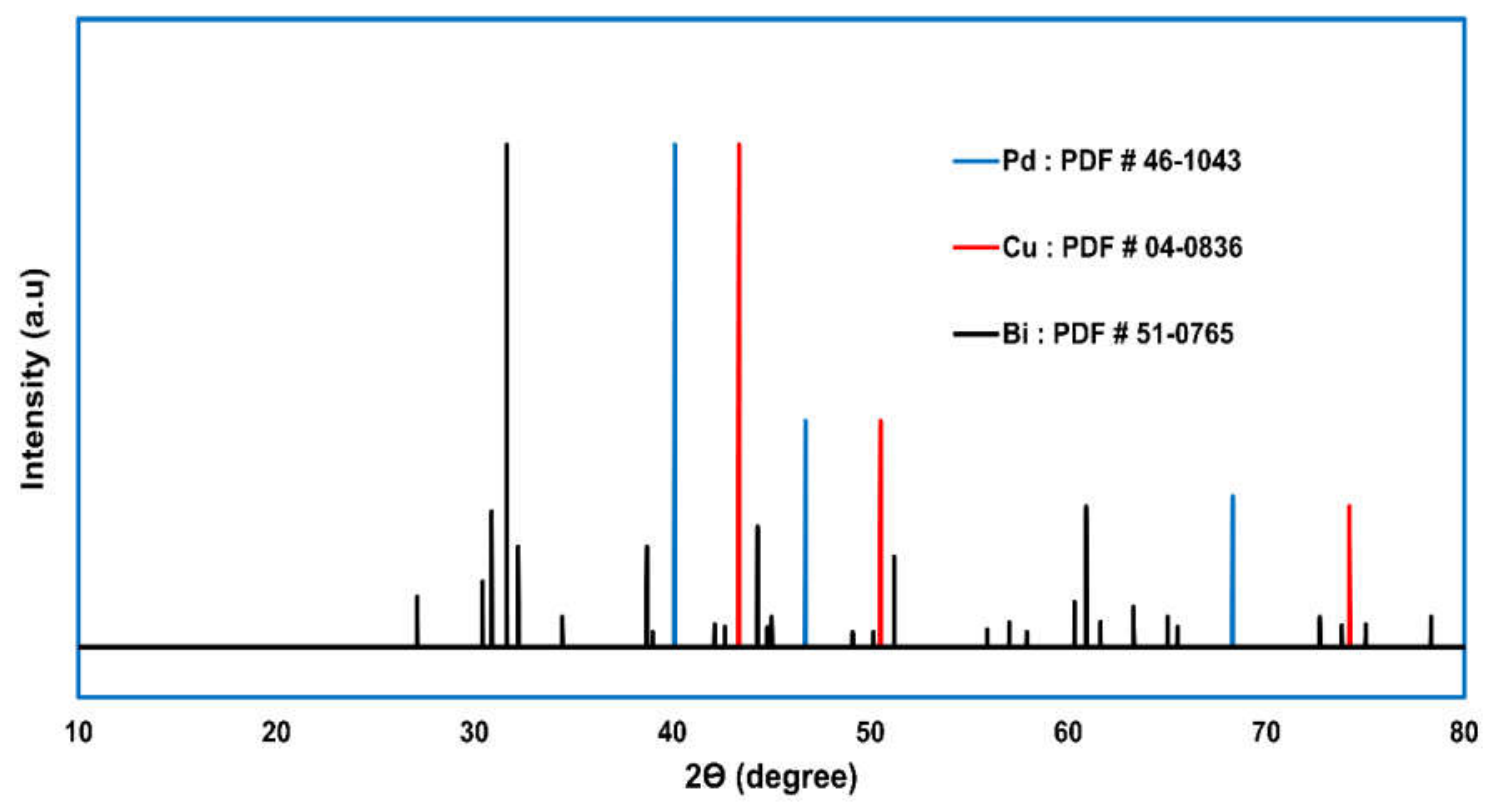

Figure S4. Standard Powder XRD patterns of metallic Pd, Bi, and Cu. 


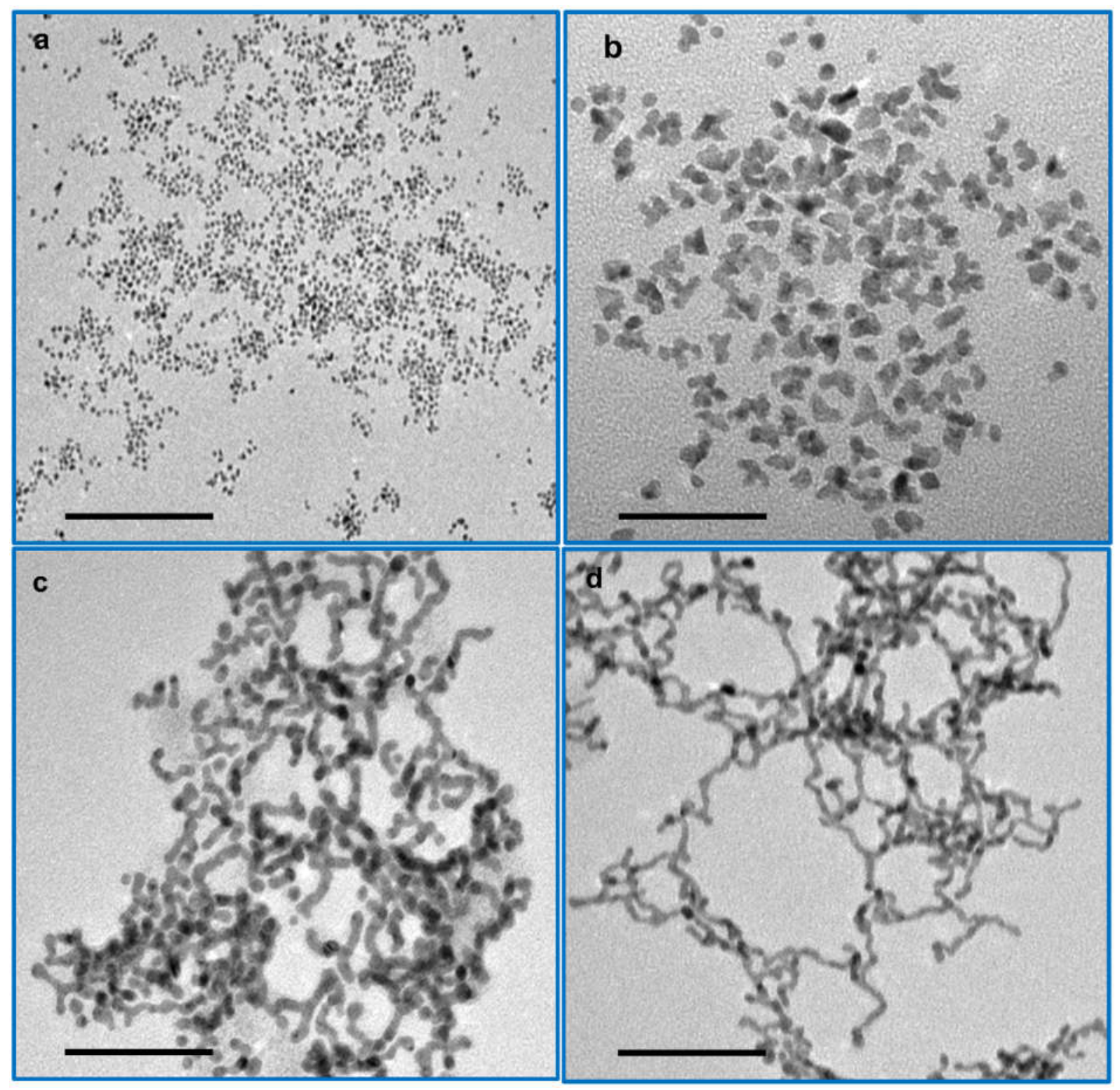

Figure S5. TEM images of the products formed at different reaction stages: (a) $30 \mathrm{sec}$, (b) 2 min,

(c) $10 \mathrm{~min}$, and (d) $30 \mathrm{~min}$. Scale bars: $50 \mathrm{~nm}$ for a, b, c, and d, respectively. 


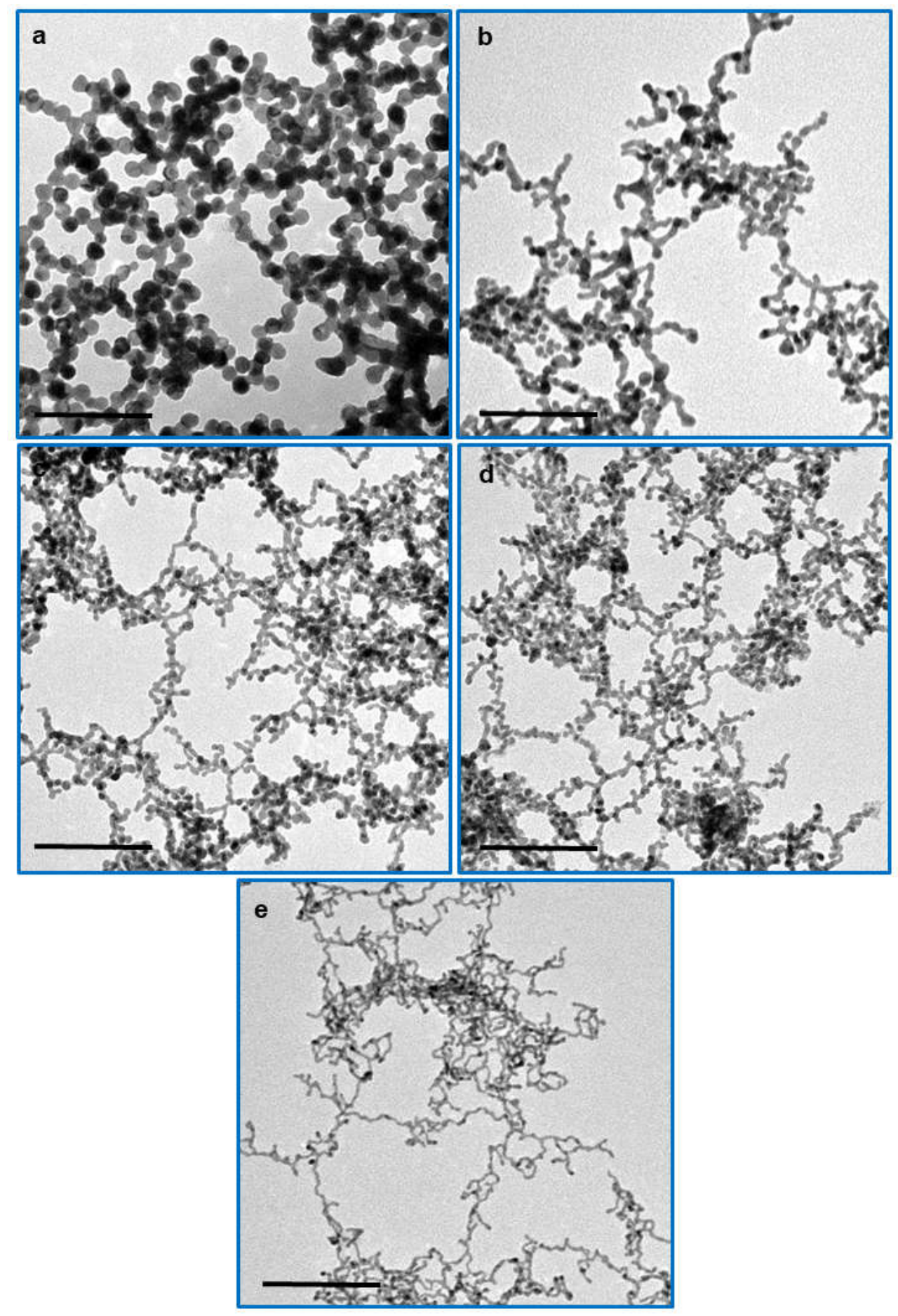

Figure S6. TEM images of the products synthesized with different $\mathrm{Mo}(\mathrm{CO})_{6}$ dosages, (a) $0 \mathrm{mg}$, (b) $2 \mathrm{mg}$, (c) $4 \mathrm{mg}$, (d) $6 \mathrm{mg}$, and (e) $10 \mathrm{mg}$. Scale bars: $50 \mathrm{~nm}$ for a, $100 \mathrm{~nm}$ for b, c, d, and e, respectively. 


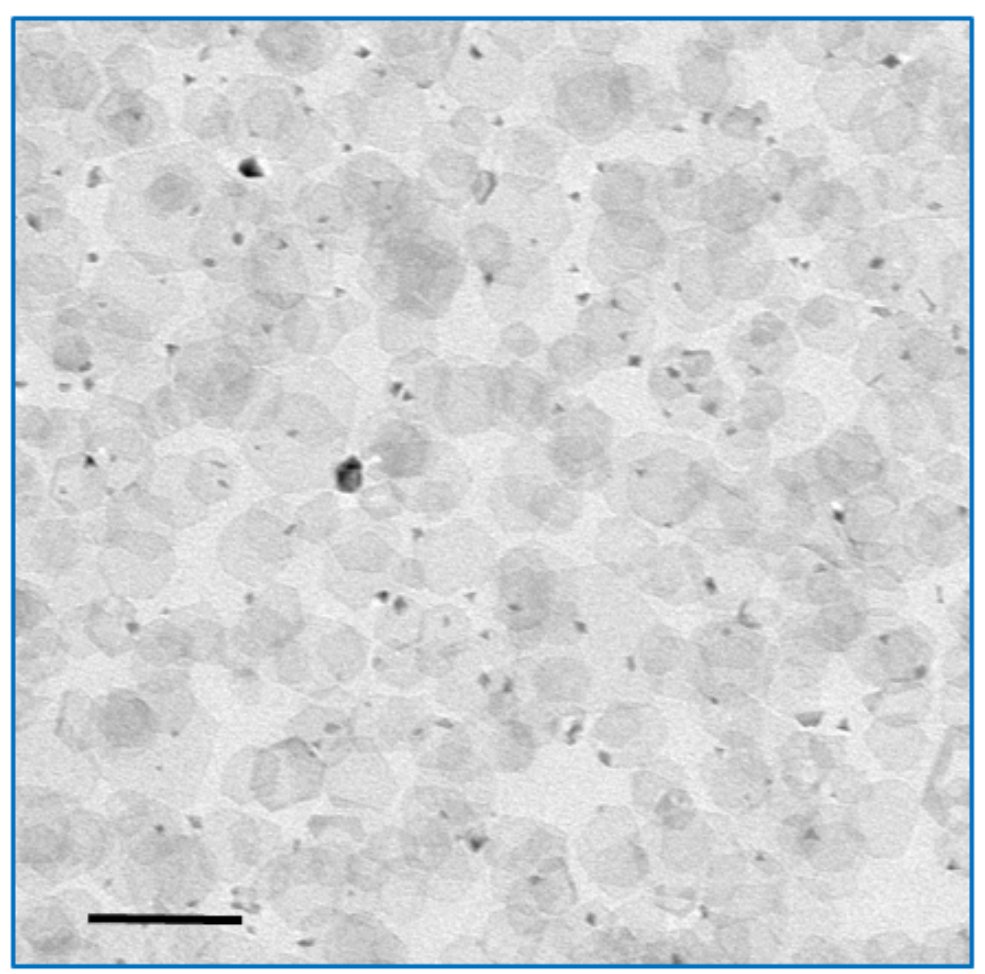

Figure S7. Pd nanosheets obtained in the absence of Bi precursor. Scale bar: $50 \mathrm{~nm}$. 

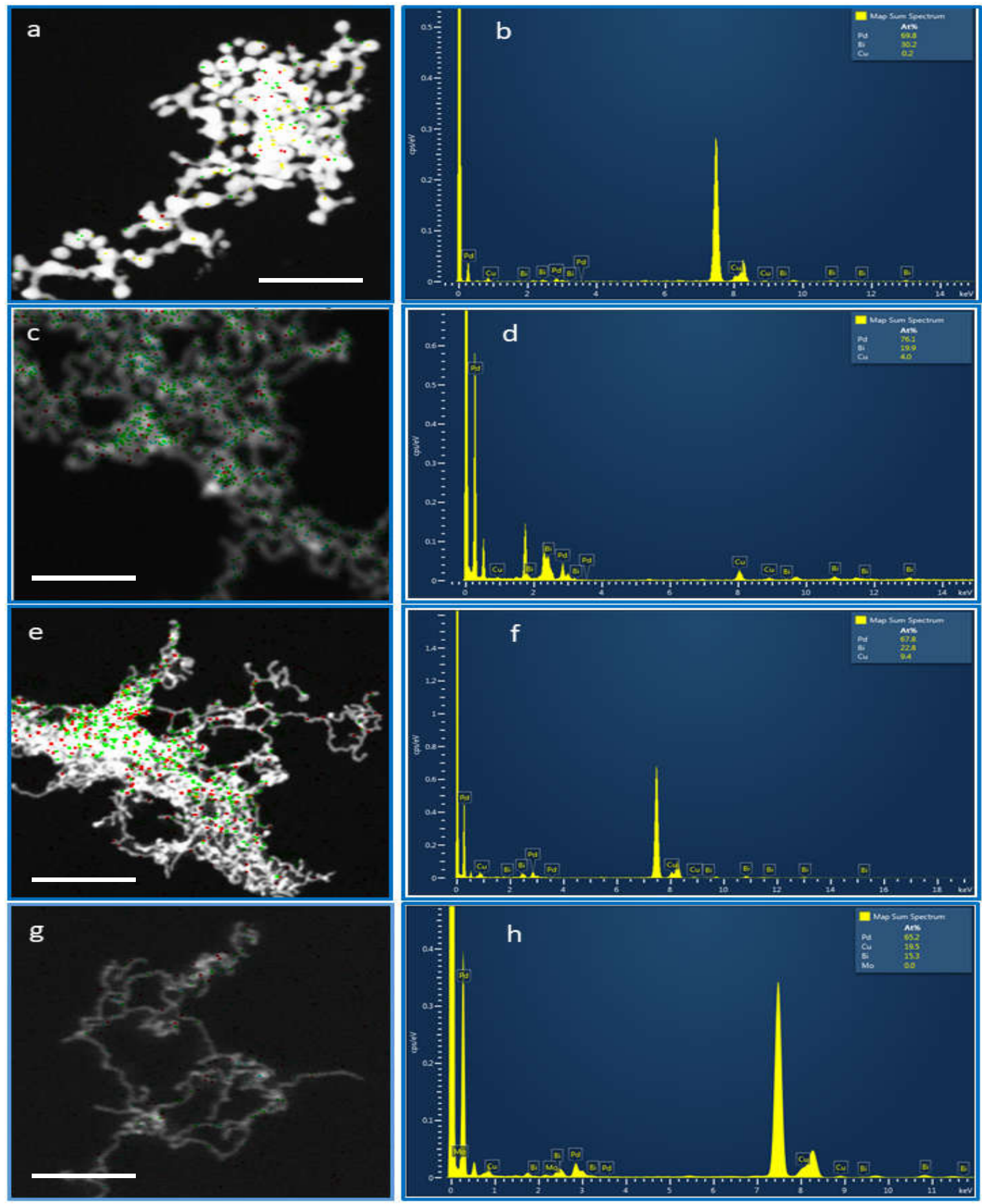

Figure S8. EDS spectra of ultrathin PdBiCu NWs formed at different reaction stages, (a, b) 1 min, (c, d) $15 \mathrm{~min}$, (e, f) $1 \mathrm{~h}$, and (g, h) $2 \mathrm{~h}$. Scale bars: $100 \mathrm{~nm}$ for a, c, e, and g, respectively. 


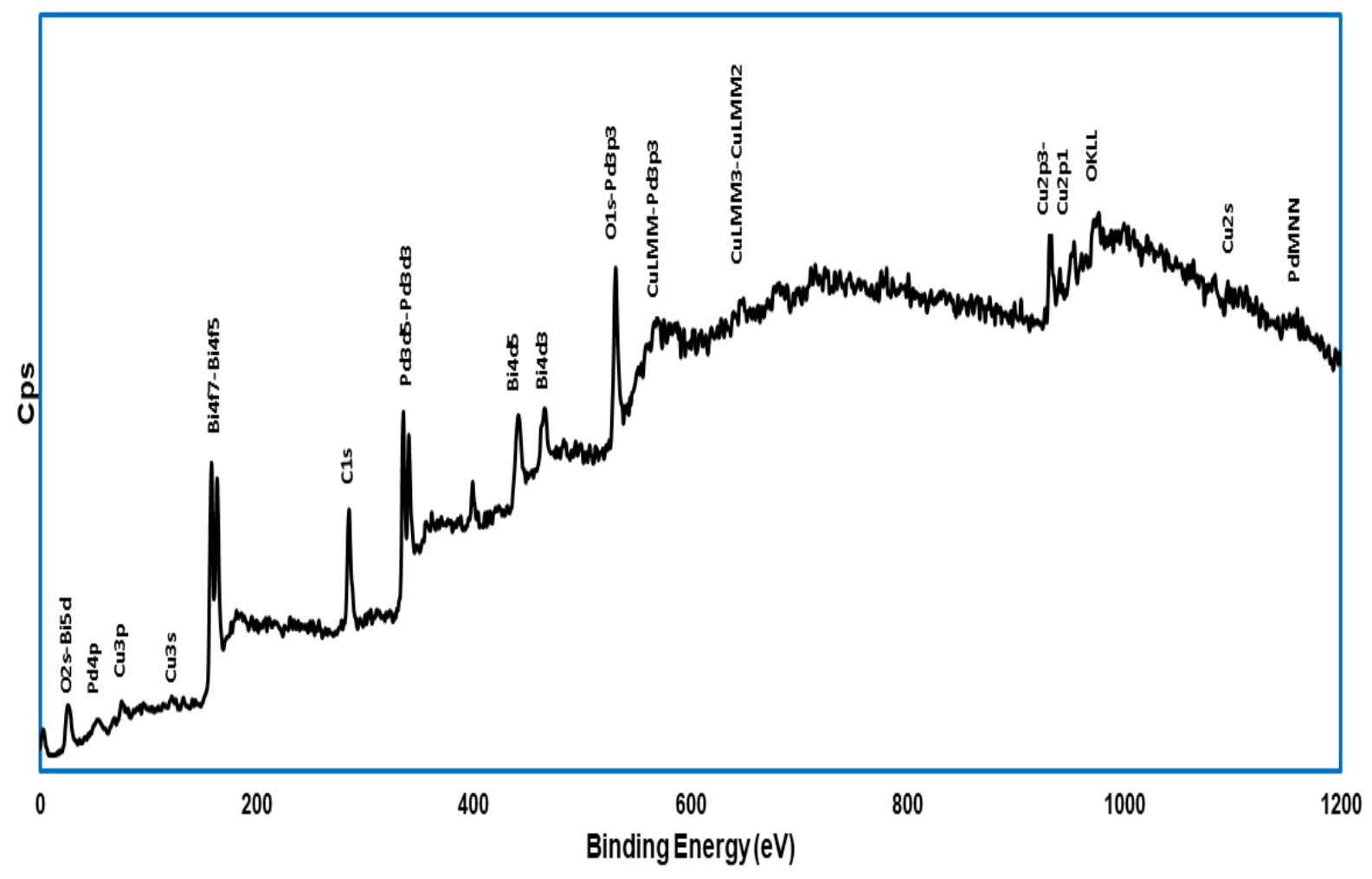

Figure S9. XPS survey spectrum of synthesized PdBiCu NWs. 


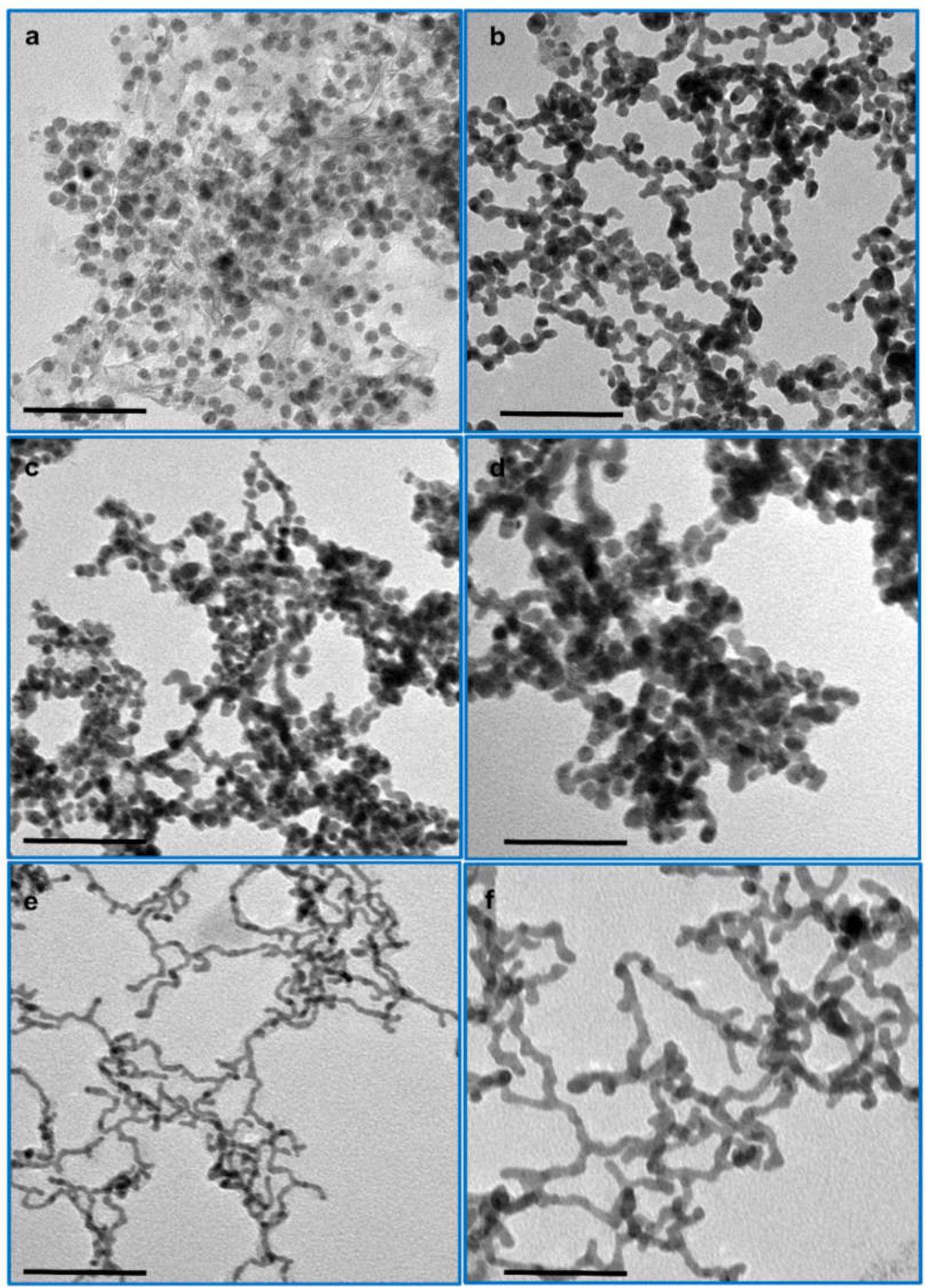

Figure S10. TEM images of the products obtained by using different surfactants and cosurfactants, (a) only PVP, (b) only TBAB, (c) PVP + TMAB, (d) PVP + TEAB, (e) PVP + TBAB, and (f) PVP + DTAB. Scale bars: $50 \mathrm{~nm}$ for a, b, c, d, e, and f, respectively. 


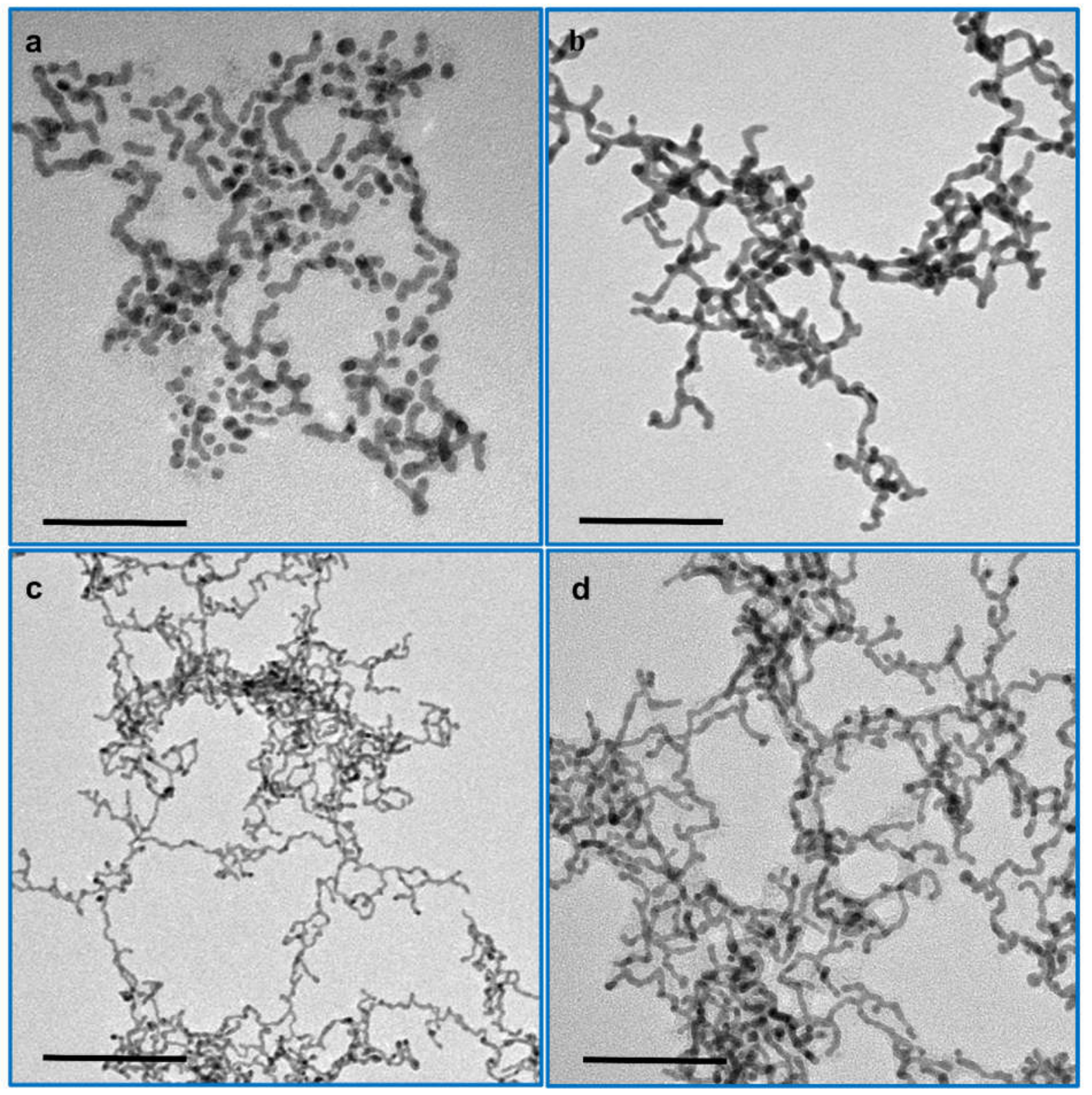

Figure S11. TEM images of the products obtained with different reaction temperatures, (a) $80{ }^{\circ} \mathrm{C}$, (b) $100{ }^{\circ} \mathrm{C}$, (c) $130{ }^{\circ} \mathrm{C}$, and (d) $150{ }^{\circ} \mathrm{C}$. Scale bars: $100 \mathrm{~nm}$ for a, b, c, and d, respectively. 


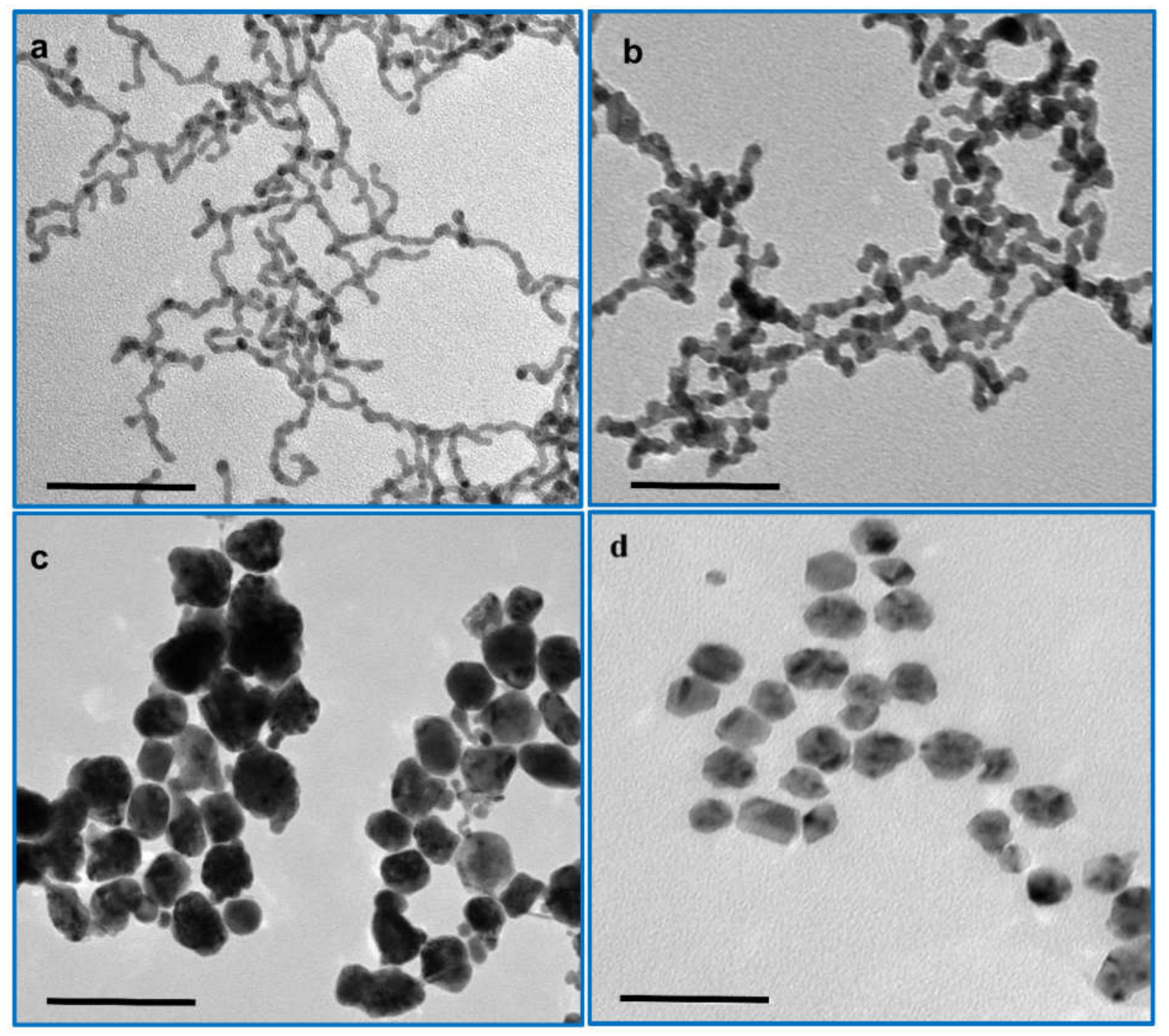

Figure S12. Effect of capping agents on the morphology of the product, (a) TBAB, (b) $\mathrm{NaCl}$, (c) Trisodium Citrate, and (d) KI. Scale bars: $100 \mathrm{~nm}$ for a and b, $50 \mathrm{~nm}$ for c and d. 


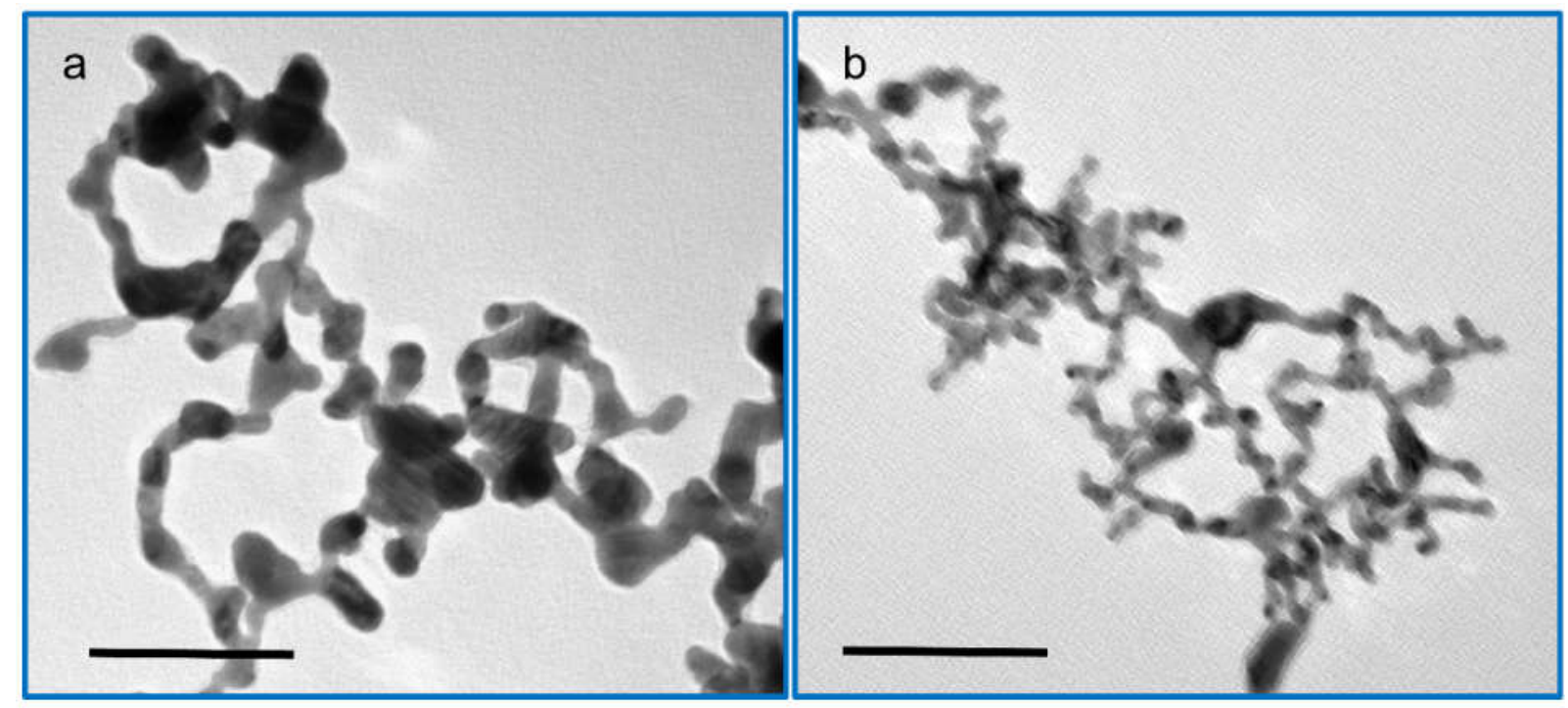

Figure S13. TEM images of the products synthesized using Formamide like solvents, (a) N, Ndimethylacetamide, (b) N, N-dimethylformamide. Scale bars: $50 \mathrm{~nm}$ for a and b, respectively. 

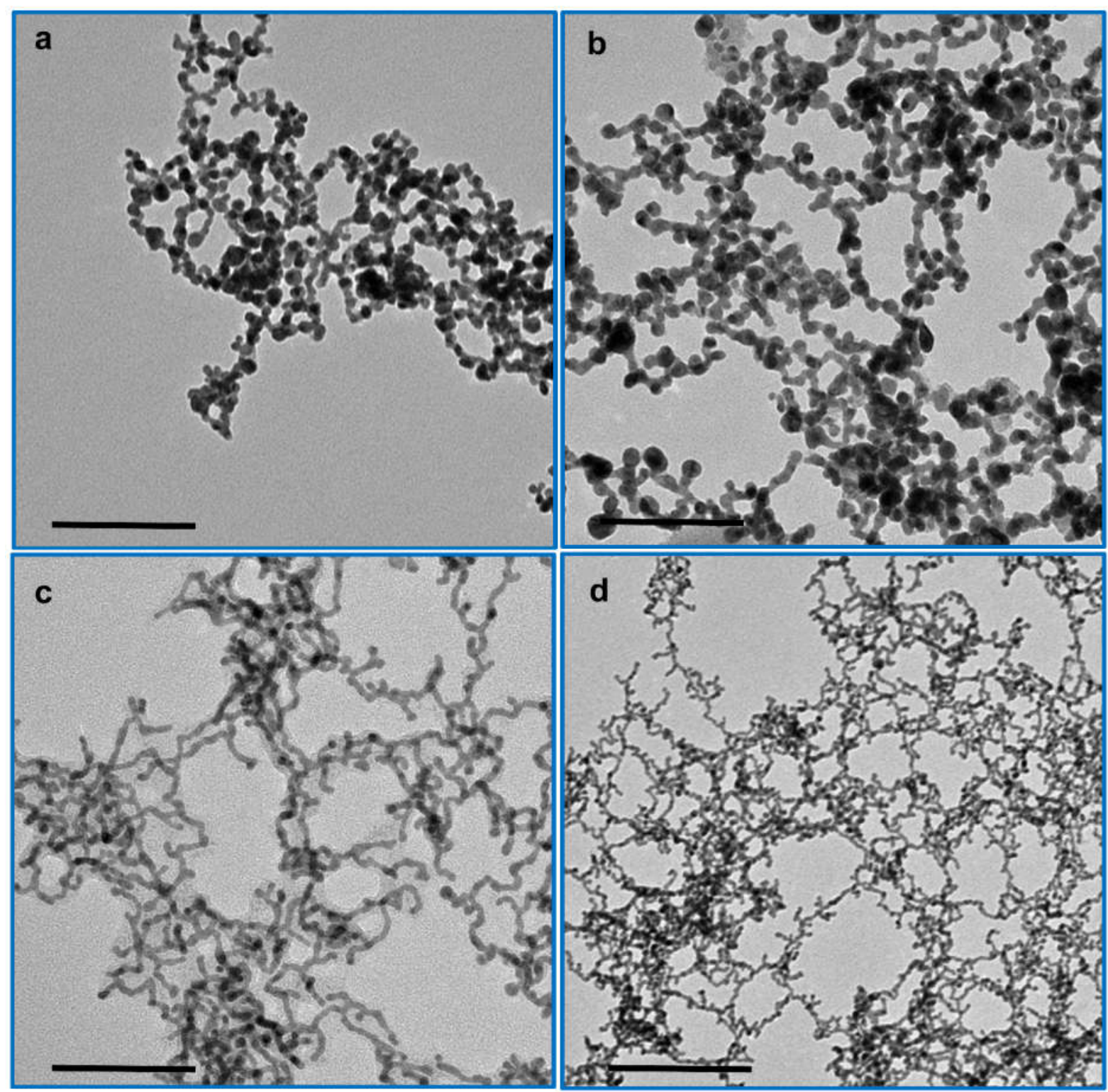

Figure S14. TEM images of the products obtained with different Copper precursors, (a) acetate, (b) sulphate, (c) acetylacetonate, and (d) chloride. Scale bars: $100 \mathrm{~nm}$ for a, b, c, and d, respectively. 

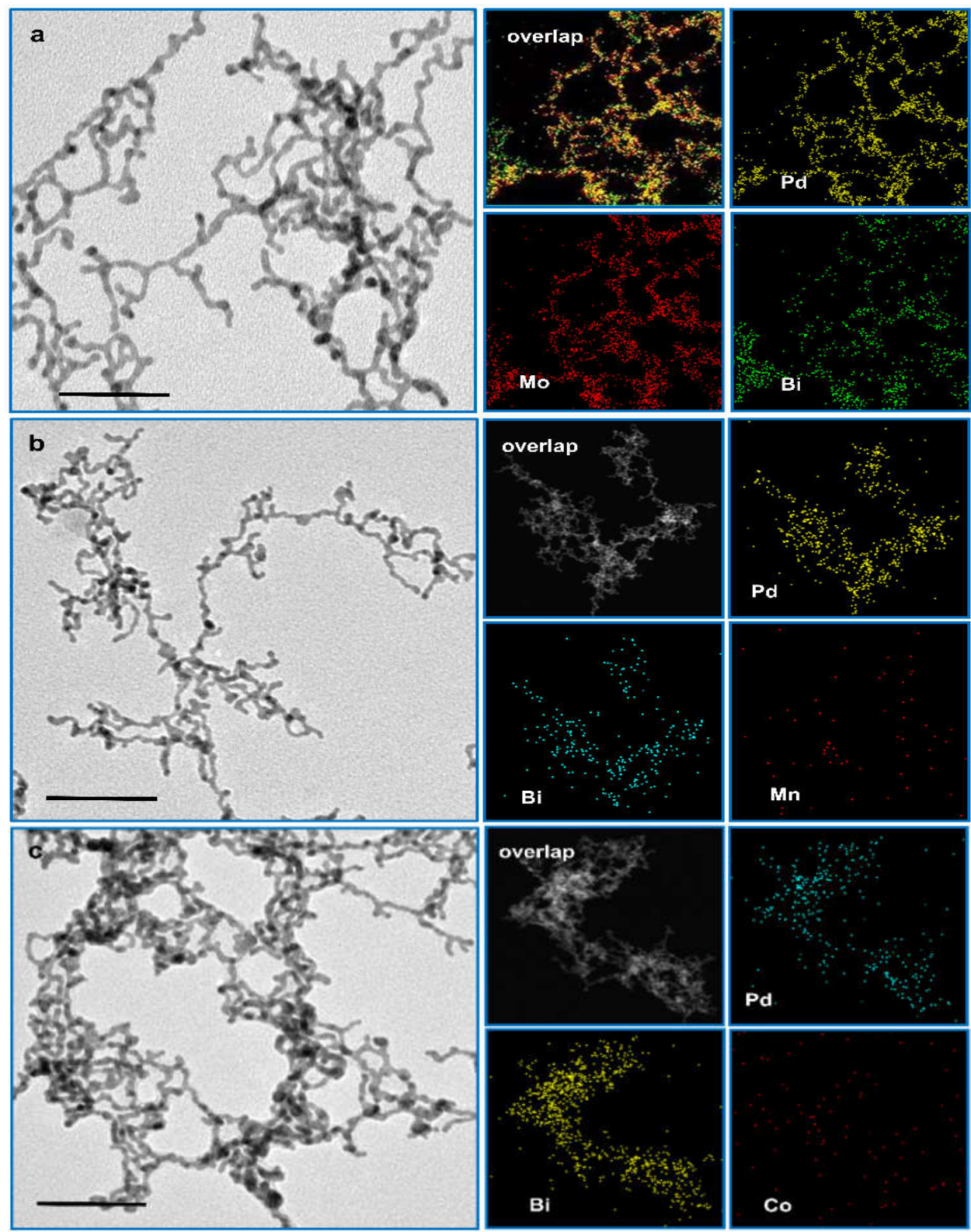

Figure S15. Extension of the synthesis protocol to the synthesis of ultrathin PdBiMo, PdBiMn, and PdBiCo NWs. TEM images and the corresponding EDS elemental mapping results of (a) ultrathin PdBiMo NWs, (b) ultrathin PdBiMn NWs, and (c) ultrathin PdBiCo NWs. Scale bars: $100 \mathrm{~nm}$ for $\mathrm{a}, \mathrm{b}$, and c, respectively. 

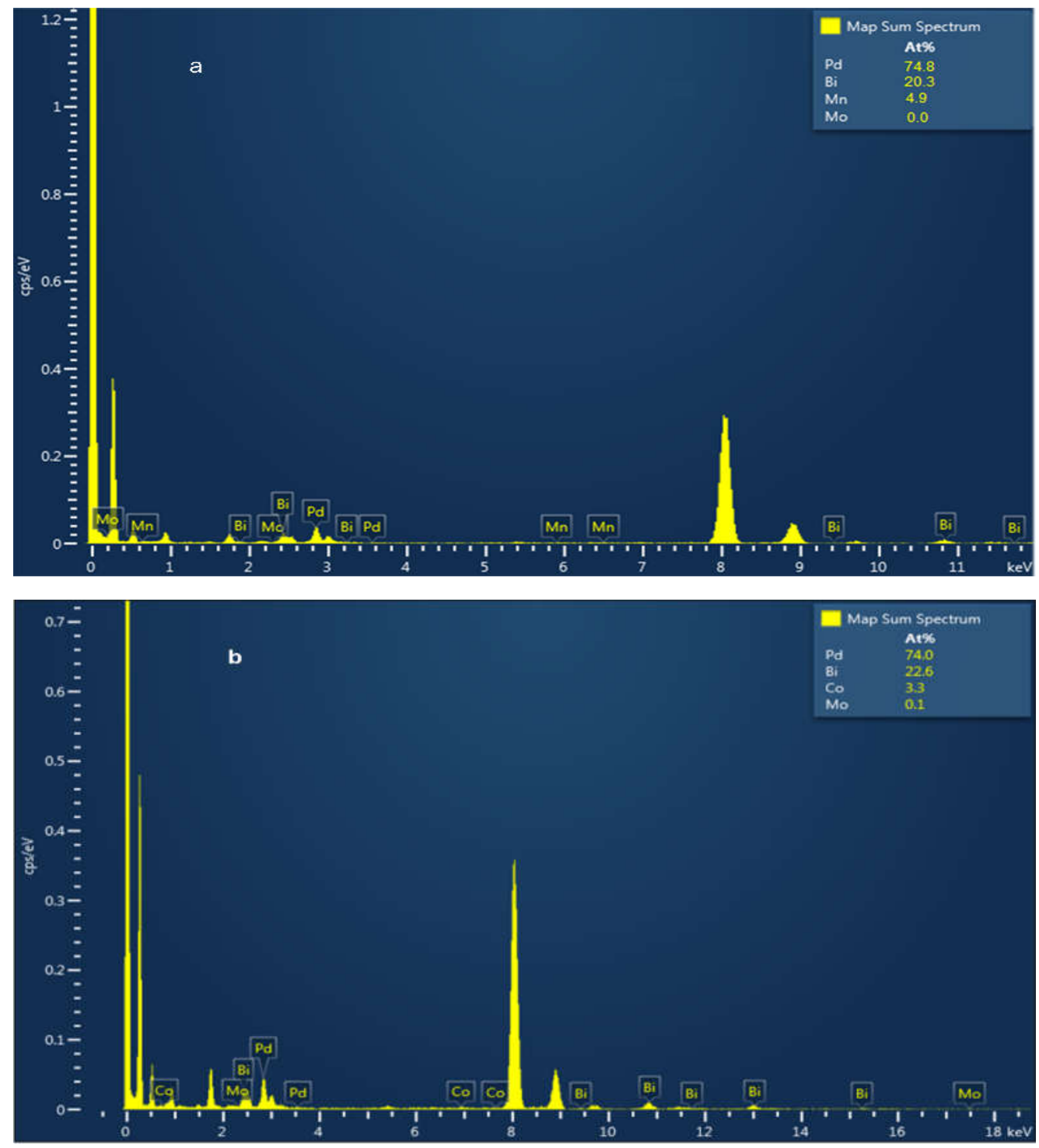

\section{Energy / KeV}

Figure S16. EDS spectra of ultrathin (a) PdBiMn NWs and (b) PdBiCo NWs. 


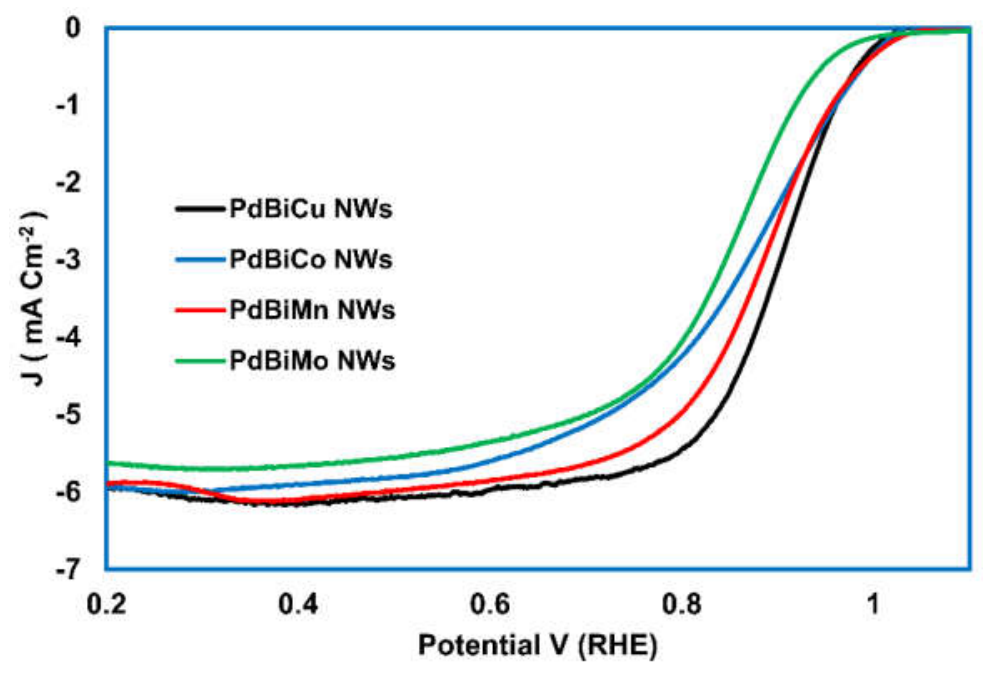

Figure S17. ORR performance of PdBiM NWs in $0.1 \mathrm{M} \mathrm{HClO}_{4}$ aqueous solution.
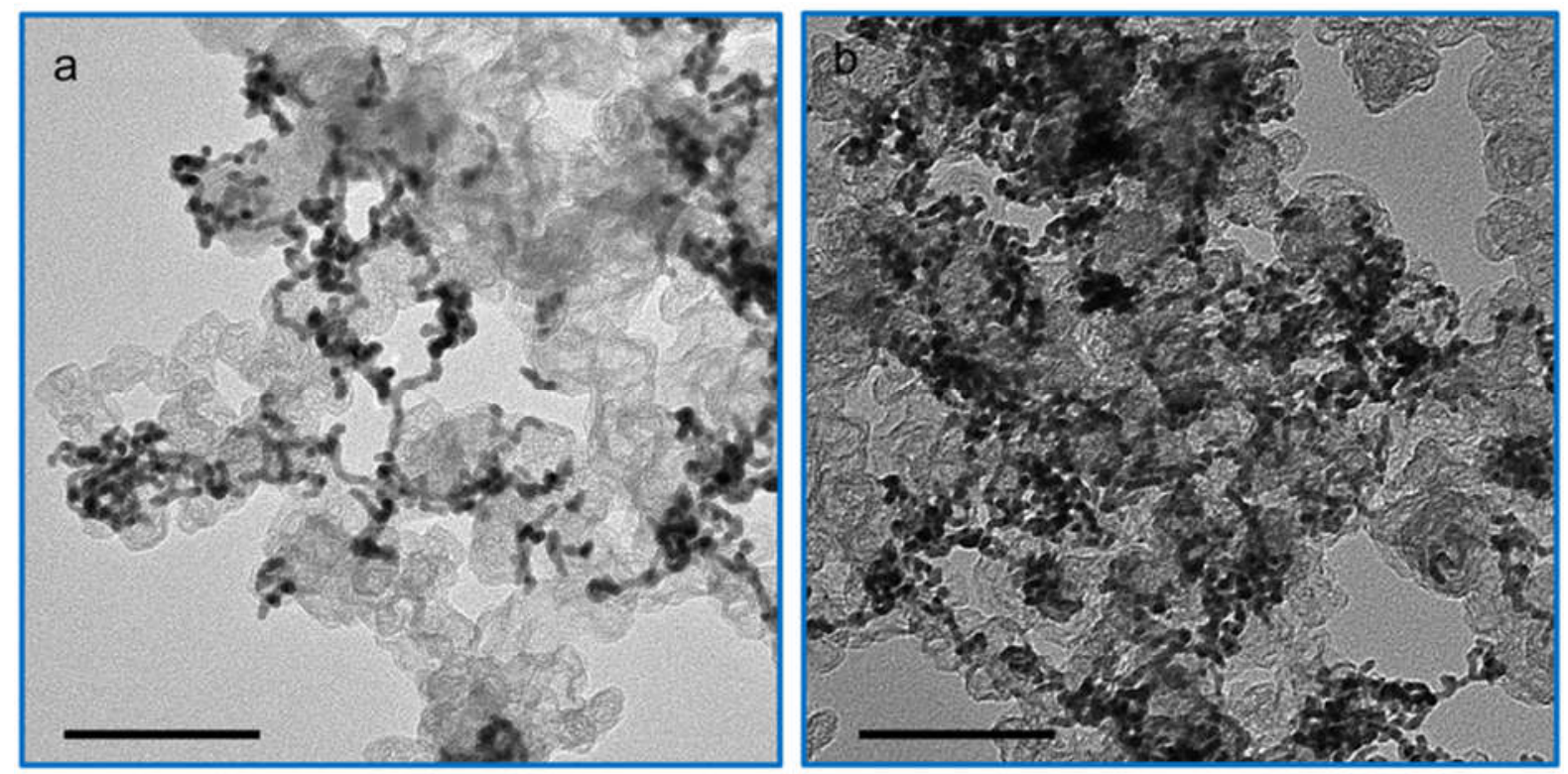

Figure S18. TEM images of the Vulcan carbon supported PdBiCu NWs (a) before and (b) after ADT test. Scale bars: $100 \mathrm{~nm}$ for a and $\mathrm{b}$, respectively. 


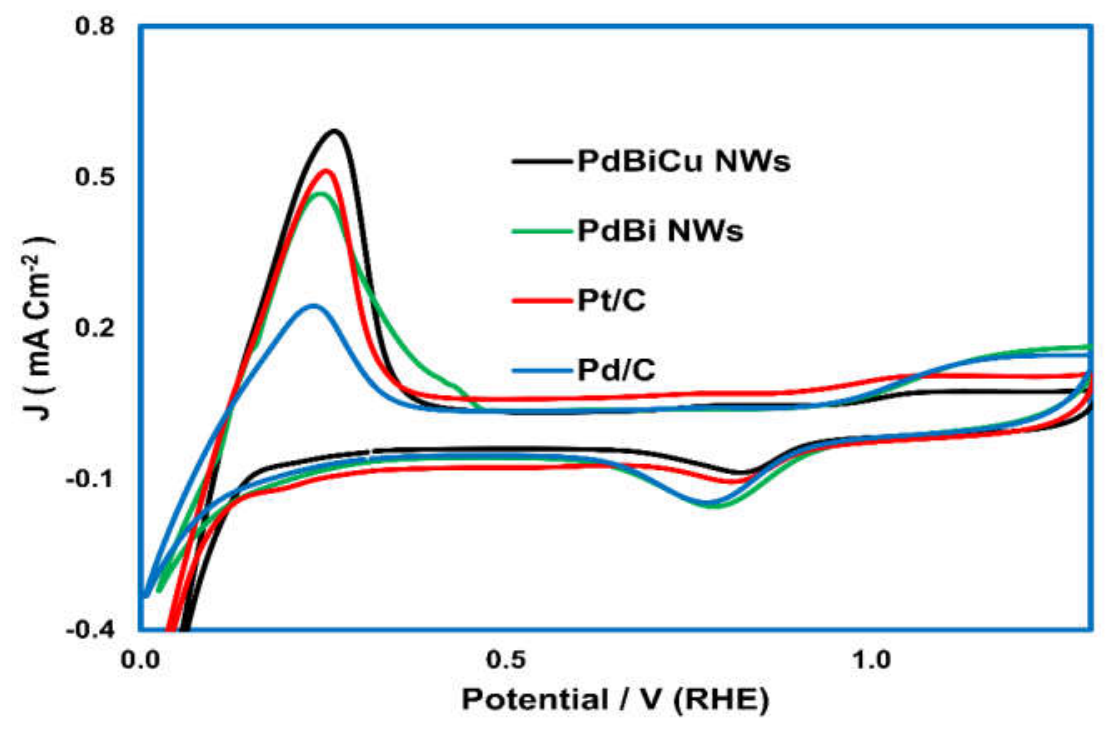

Figure S19. Cyclic voltammograms (CV) of the as-synthesized PdBiCu NWs, PdBi NWs, Pd/C, and $\mathrm{Pt} / \mathrm{C}$ in $0.1 \mathrm{M} \mathrm{HClO}_{4}$ aqueous solution at a scan rate of $50 \mathrm{mV} \mathrm{s}^{-1}$.
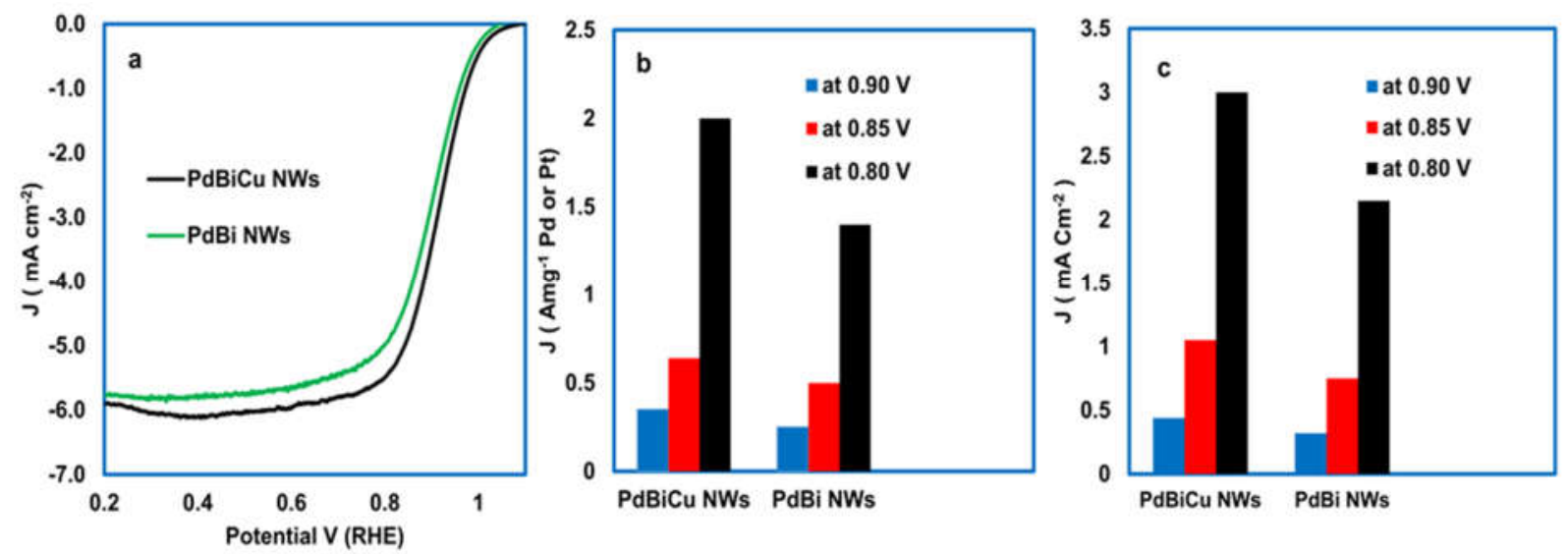

Figure S20. (a) LSV plots of PdBiCu NWs and PdBi NWs, (b) the corresponding mass activity and (c) specific activity. 

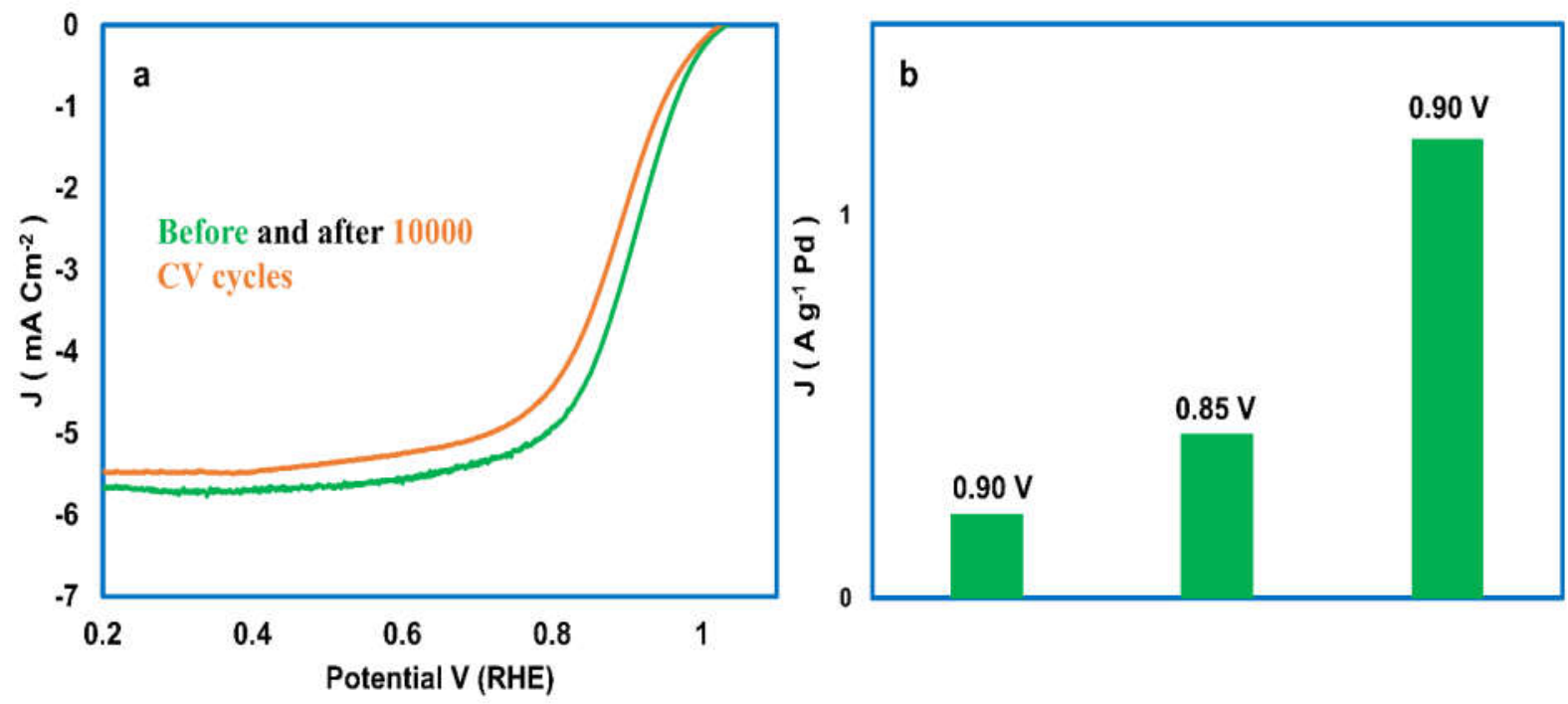

Figure S21. (a) Durability performance and (b) the corresponding mass activity of PdBi NWs.
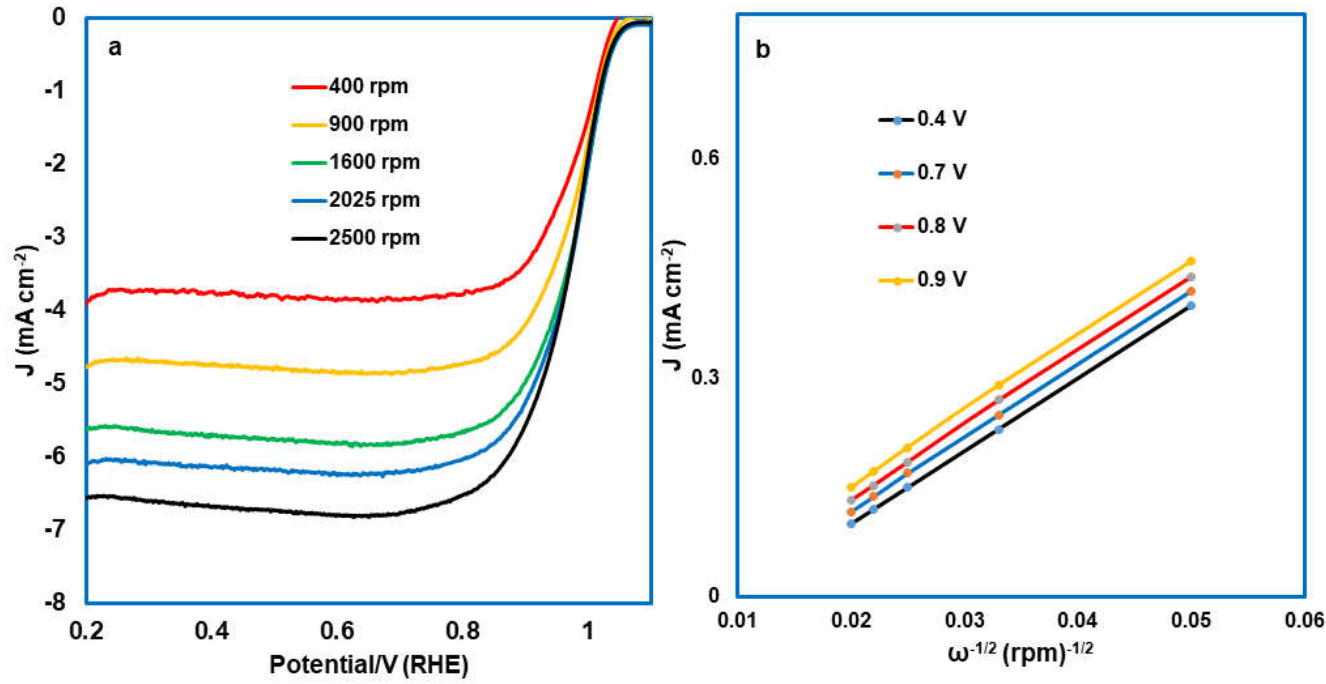

Figure S22. (a) LSV curves (different rotation speeds) and (b) the corresponding K-L plots (different potentials) of $\mathrm{PdBiCu}_{\mathrm{NWs}}$ measured in $0.1 \mathrm{M} \mathrm{HClO}_{4}$ aqueous solution. 

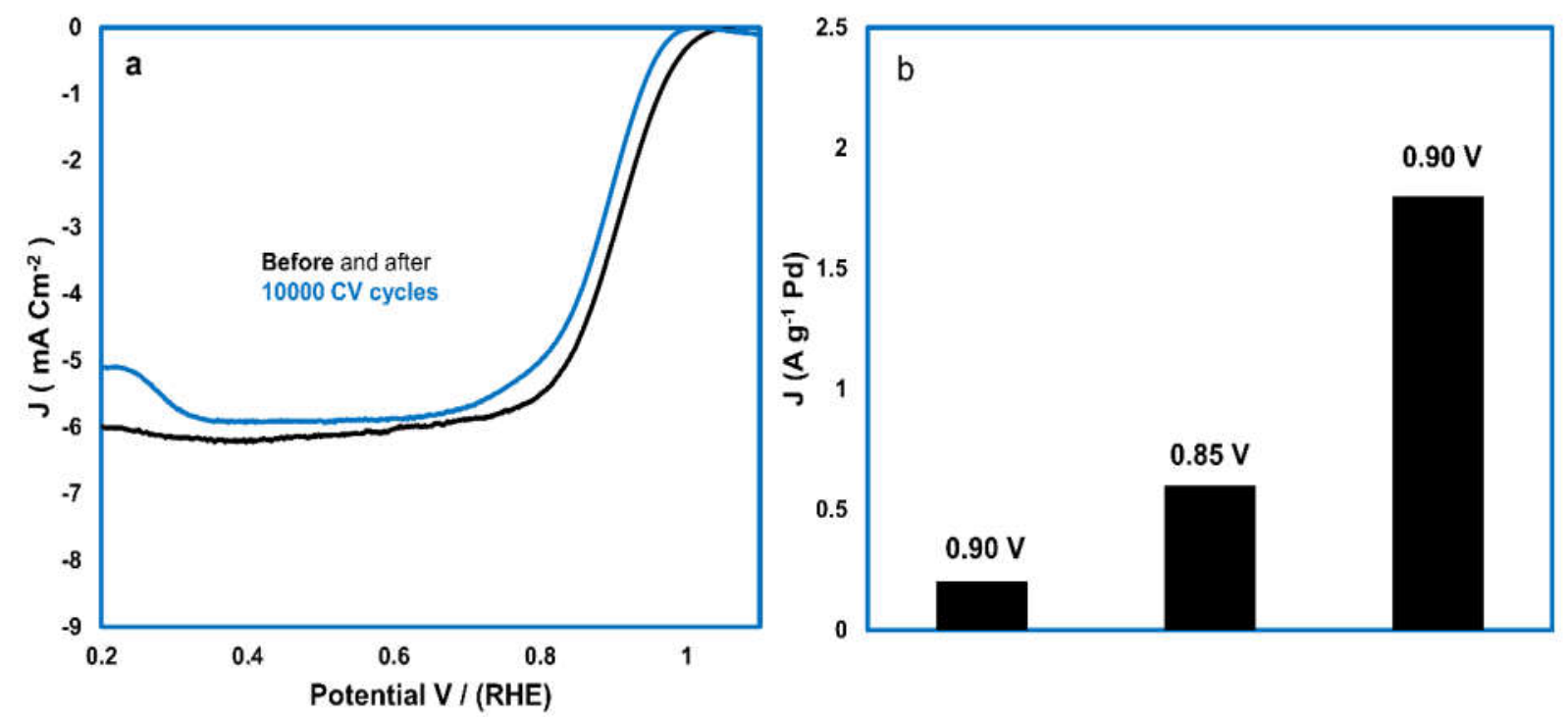

Figure S23. (a) Durability performance and (b) the corresponding mass activity of synthesized PdBiCu NWs.
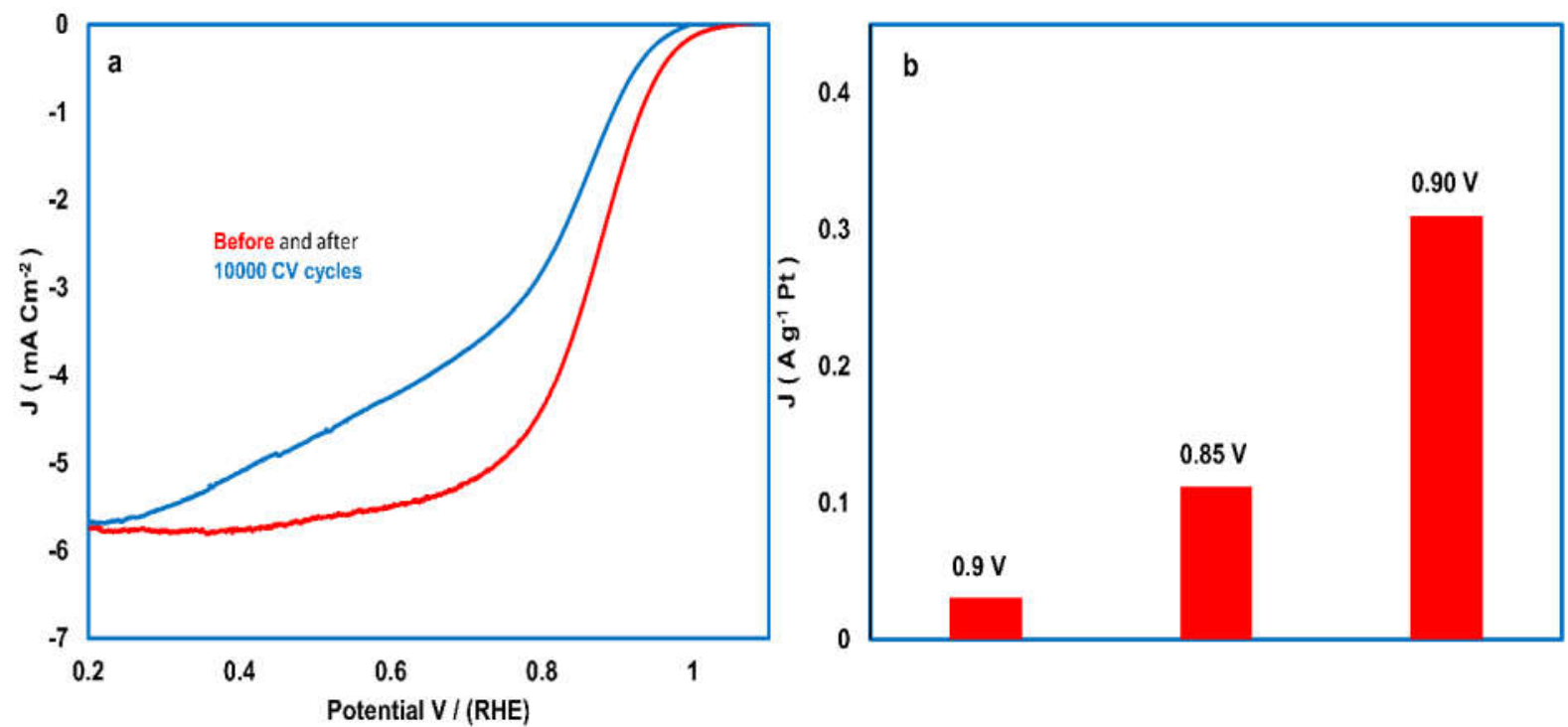

Figure S24. (a) Durability performance and (b) the corresponding mass activity of Pt/C catalyst. 

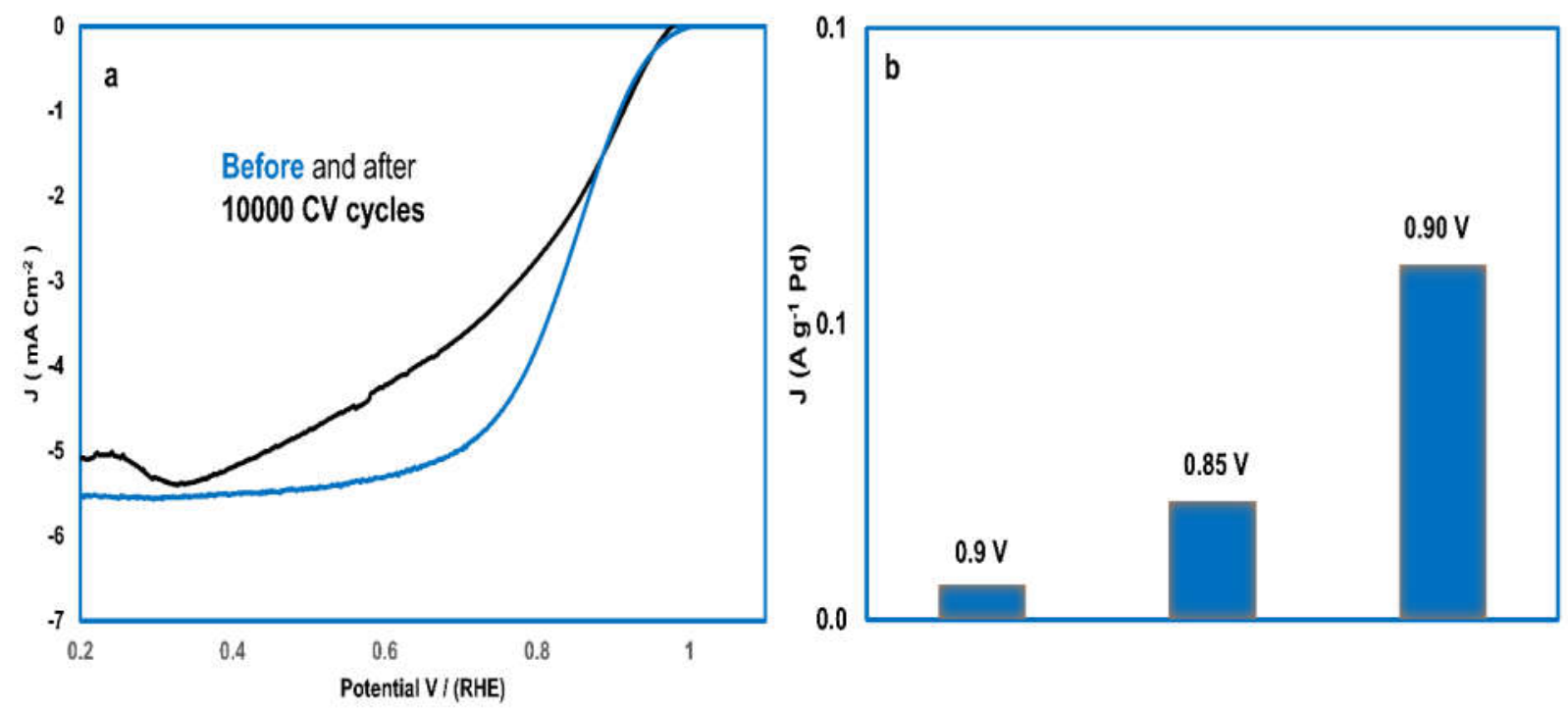

Figure S25. (a) Durability performance and (b) the corresponding mass activity of $\mathrm{Pd} / \mathrm{C}$ catalyst.
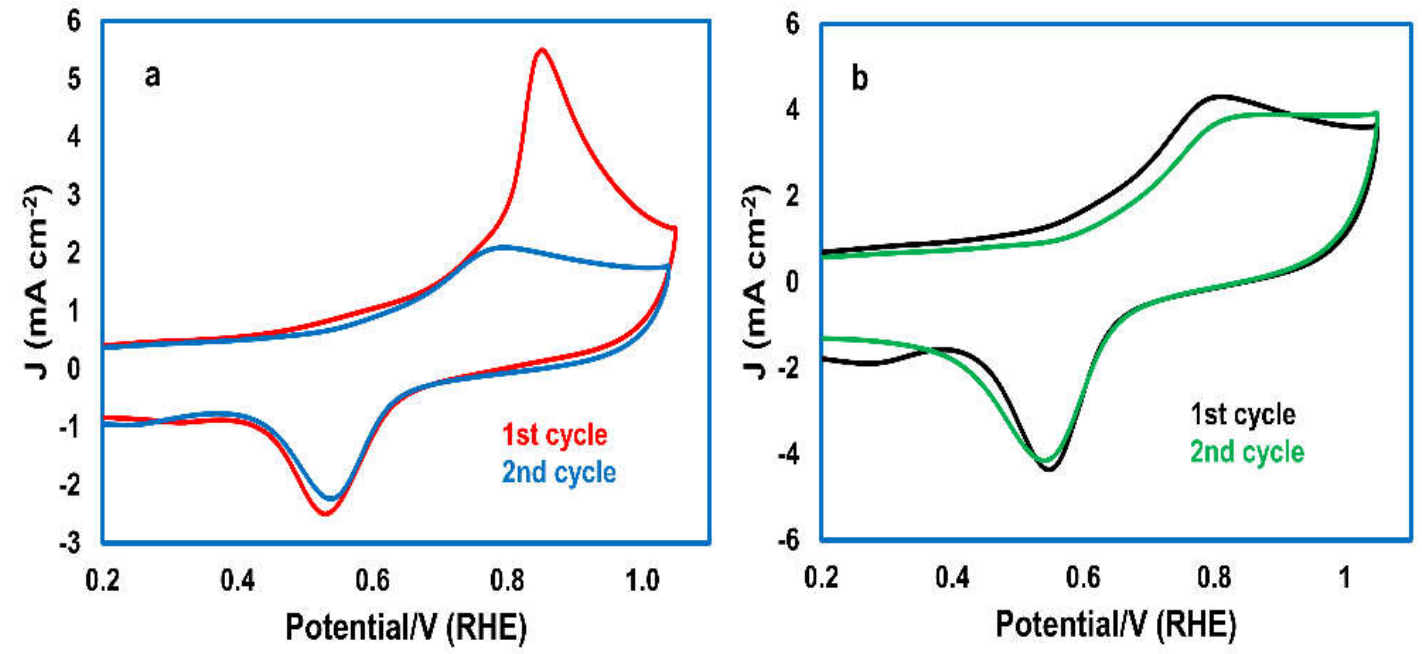

Figure S26. CO tolerance test for (a) $\mathrm{Pd} / \mathrm{C}$ and (b) PdBiCu NWs. 

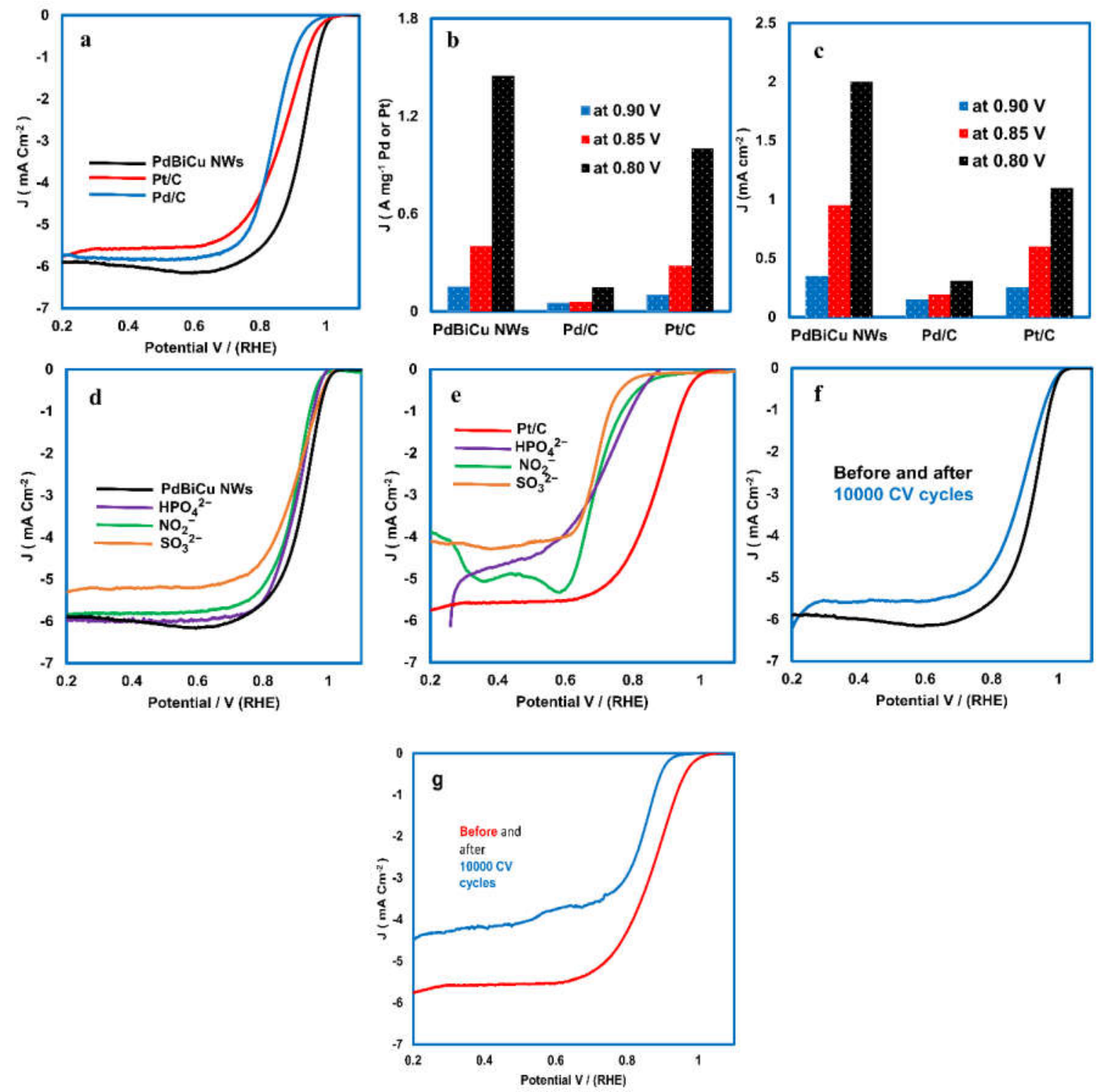

Figure S27. (a) ORR polarization curves (1600 rpm in $0.1 \mathrm{M} \mathrm{KOH})$ and the corresponding (b) mass activity and (c) specific activity of $\mathrm{PdBiCu} \mathrm{NWs}, \mathrm{Pt} / \mathrm{C}$, and $\mathrm{Pd} / \mathrm{C}$ catalysts. (d, e) Antipoisoning and (f, g) durability tests for (d, f) PdBiCu NWs and (e, g) Pt/C. 
Supplementary Table S1. ICP-OES data of the synthesized PdBiCu NWs.

\begin{tabular}{|c|c|c|c|c|c|}
\hline Sample & Element & at\% & $\begin{array}{c}\text { After acid } \\
\text { treatment } \\
\text { at } \%\end{array}$ & \multicolumn{2}{|c|}{$\begin{array}{c}\text { After 10000 CV cycles } \\
\text { at } \%\end{array}$} \\
\hline \multirow{3}{*}{ PdBiCu NWs } & $\mathrm{Pd}$ & 65 & 73 & $\mathrm{Pd}$ & 76 \\
& $\mathrm{Bi}$ & 15 & 14 & $\mathrm{Bi}$ & 16 \\
& $\mathrm{Cu}$ & 20 & 13 & $\mathrm{Cu}$ & 8 \\
\hline
\end{tabular}

Supplementary Table S2. Electrochemical parameters of the synthesized and commercial catalysts.

\begin{tabular}{|c|c|c|c|c|c|}
\hline Catalyst & $\begin{array}{l}\text { Working } \\
\text { Electrolyte }\end{array}$ & $\begin{array}{l}\text { Half Wave } \\
\text { Potential } \\
\left(\mathrm{E}_{1 / 2}\right)\end{array}$ & $\begin{array}{l}\text { Electrochemical } \\
\text { Surface Area } \\
\left(\mathrm{m}^{2} \mathrm{~g}^{-1}\right)\end{array}$ & $\begin{array}{l}\text { Specific Activity } \\
\qquad\left(\mathrm{mA} \mathrm{cm}^{-2}\right)\end{array}$ & $\begin{array}{l}\text { Mass Activity } \\
\quad\left(\mathrm{A} \mathrm{mg}^{-1}\right)\end{array}$ \\
\hline $\begin{array}{c}\mathrm{Pd} / \mathrm{C} \\
\text { (commercial) }\end{array}$ & $0.1 \mathrm{M} \mathrm{HClO}_{4}$ & $0.85 \mathrm{~V}$ & 40 & $\begin{array}{c}0.03(0.9 \mathrm{~V} \text { vs } \\
\text { RHE) } \\
0.28(0.8 \mathrm{~V} \text { vs } \\
\text { RHE })\end{array}$ & $\begin{array}{c}0.012(0.9 \mathrm{~V} \text { vs RHE }) \\
0.11(0.8 \mathrm{~V} \text { vs RHE })\end{array}$ \\
\hline $\begin{array}{c}\mathrm{Pt} / \mathrm{C} \\
\text { (commercial) }\end{array}$ & $0.1 \mathrm{M} \mathrm{HClO}_{4}$ & $0.86 \mathrm{~V}$ & 65 & $\begin{array}{c}0.17(0.9 \mathrm{~V} \text { vs } \\
\text { RHE }) \\
1.70(0.8 \mathrm{~V} \text { vs } \\
\text { RHE })\end{array}$ & $\begin{array}{c}0.11(0.9 \mathrm{~V} \text { vs RHE }) \\
1.1(0.8 \mathrm{~V} \text { vs RHE }\end{array}$ \\
\hline PdBi NWs & $0.1 \mathrm{M} \mathrm{HClO}_{4}$ & 0.89 & 65 & $\begin{array}{c}2.15(0.8 \mathrm{~V} \text { vs } \\
\text { RHE) }\end{array}$ & $\begin{array}{c}0.25(0.9 \text { v vs RHE }) \\
1.40(0.8 \text { vs RHE })\end{array}$ \\
\hline $\mathrm{PdBiCu}$ NWs & $0.1 \mathrm{M} \mathrm{HClO}_{4}$ & $0.91 \mathrm{~V}$ & 67 & $\begin{array}{c}0.53(0.9 \mathrm{~V} \text { vs } \\
\text { RHE }) \\
3 \text { (0.8 V vs RHE) }\end{array}$ & $\begin{array}{c}0.350(0.9 \mathrm{~V} \text { vs RHE }) \\
2.0(0.8 \mathrm{~V} \text { vs RHE })\end{array}$ \\
\hline
\end{tabular}


Supplementary Table S3. Literature summary of specific and mass activities of Pd based catalysts for oxygen reduction reaction (ORR).

\begin{tabular}{|c|c|c|c|c|}
\hline Catalyst & $\begin{array}{l}\text { Working } \\
\text { Solution }\end{array}$ & $\begin{array}{l}\text { Specific Activity } \\
\mathrm{mA} \mathrm{cm}^{-2}\end{array}$ & $\begin{array}{l}\text { Mass Activity } \\
\mathrm{A} \mathrm{mg}^{-1}\end{array}$ & \\
\hline $\begin{array}{c}\mathrm{Rh}_{8} \mathrm{Pd}_{92} \\
\text { Octahedron }\end{array}$ & $0.1 \mathrm{M} \mathrm{HClO}_{4}$ & NA & $0.26(0.9 \mathrm{~V}$ vs RHE) & 1 \\
\hline $\begin{array}{c}\mathrm{Rh}_{20} \mathrm{Pd}_{80} / \mathrm{C} \\
\text { Nano dendrites }\end{array}$ & $0.1 \mathrm{M} \mathrm{KOH}$ & NA & 0.48 (0.8 V vs RHE) & 2 \\
\hline $\begin{array}{l}\mathrm{PdCu} \mathrm{B}_{2} \text { phase } \\
\text { Nanoparticle }\end{array}$ & $0.1 \mathrm{M} \mathrm{HClO}_{4}$ & 0.168 (0.9 V vs RHE) & 0.0415 (0.9 V vs RHE) & 3 \\
\hline $\begin{array}{l}\text { Pd tetrahedron } \\
/ / \mathrm{W}_{18} \mathrm{O}_{49}\end{array}$ & $0.1 \mathrm{M} \mathrm{KOH}$ & 0.45 (0.9 V vs RHE) & 0.216 (0.9 V vs RHE) & 4 \\
\hline $\begin{array}{c}\text { Nanoporous } \\
\text { PdNi }\end{array}$ & $0.1 \mathrm{M} \mathrm{KOH}$ & NA & 0.4 (0.85 V vs RHE) & 5 \\
\hline $\mathrm{Cu}_{3} \mathrm{Pd} / \mathrm{Graphene}$ & $0.1 \mathrm{M} \mathrm{KOH}$ & NA & 0.045 (0.75 V vs RHE) & 6 \\
\hline $\begin{array}{c}\text { PdCuCo } \\
\text { anisotropic } \\
\text { structure }\end{array}$ & $0.1 \mathrm{M} \mathrm{HClO}_{4}$ & $\begin{array}{c}0.252 \text { (0.9 V vs RHE) } \\
1.7 \text { (0.8 V vs RHE) }\end{array}$ & $\begin{array}{l}0.178 \text { (0.9 V vs RHE) } \\
1.135 \text { ( } 0.8 \text { V vs RHE) }\end{array}$ & 7 \\
\hline $\begin{array}{c}\text { Ordered } \\
\mathrm{PdCuCo} / \mathrm{C} \\
\text { Nanoparticle }\end{array}$ & $0.1 \mathrm{M} \mathrm{NaOH}$ & NA & $\begin{array}{l}0.13(0.9 \mathrm{~V} \text { vs RHE }) \\
0.37(0.875 \mathrm{~V} \text { vs } \mathrm{RHE})\end{array}$ & 8 \\
\hline $\mathrm{Pd}-\mathrm{Co} / \mathrm{gCN}$ & $1 \mathrm{M} \mathrm{H}_{2} \mathrm{SO}_{4}$ & 5.8 (0.65 V vs RHE) & $\mathrm{NA}$ & 9 \\
\hline $\begin{array}{l}\mathrm{Pd}_{0.90} \mathrm{Ni}_{0.10} \\
\text { Nanowire }\end{array}$ & $0.1 \mathrm{M} \mathrm{HClO}_{4}$ & 1.96 (0.8 V vs RHE) & NA & 10 \\
\hline
\end{tabular}




\section{Supplementary References}

(1) Yan, Y.; Zhan, F.; Du, J.; Jiang, Y.; Jin, C.; Fu, M.; Zhang, H.; Yang, D., KineticallyControlled Growth of Cubic and Octahedral Rh-Pd Alloy Oxygen Reduction Electrocatalysts with High Activity and Durability. Nanoscale 2015, 7, 301-307.

(2) Qi, Y.; Wu, J.; Zhang, H.; Jiang, Y.; Jin, C.; Fu, M.; Yang, H.; Yang, D., Facile Synthesis of Rh-Pd Alloy Nanodendrites as Highly Active and Durable Electrocatalysts for Oxygen Reduction Reaction. Nanoscale 2014, 6, 7012-7018.

(3) Wang, C.; Chen, D. P.; Sang, X.; Unocic, R. R.; Skrabalak, S. E., Size-Dependent Disorder-Order Transformation in the Synthesis of Monodisperse Intermetallic $\mathrm{PdCu}$ Nanocatalysts. ACS Nano 2016, 10, 6345-6353.

(4) Lu, Y.; Jiang, Y.; Gao, X.; Wang, X.; Chen, W., Strongly Coupled Pd Nanotetrahedron/Tungsten Oxide Nanosheet Hybrids with Enhanced Catalytic Activity and Stability as Oxygen Reduction Electrocatalysts. J. Am.chem. Soc. 2014, 136, 11687-11697.

(5) Chen, L.; Guo, H.; Fujita, T.; Hirata, A.; Zhang, W.; Inoue, A.; Chen, M., Nanoporous PdNi Bimetallic Catalyst with Enhanced Electrocatalytic Performances for Electro-Oxidation and Oxygen Reduction Reactions. Adv. Funct. Mater. 2011, 21, 4364-4370.

(6) Zheng, Y.; Zhao, S.; Liu, S.; Yin, H.; Chen, Y.-Y.; Bao, J.; Han, M.; Dai, Z., ComponentControlled Synthesis and Assembly of $\mathrm{Cu}-\mathrm{Pd}$ Nanocrystals on Graphene for Oxygen Reduction Reaction. ACS appl. Mater. \& interfaces 2015, 7, 5347-5357.

(7) Zuo, Y.; Rao, D.; Li, S.; Li, T.; Zhu, G.; Chen, S.; Song, L.; Chai, Y.; Han, H., Atomic Vacancies Control of Pd-Based Catalysts for Enhanced Electrochemical Performance. Adv. Mater. 2018, 30, 1704171.

(8) Jiang, K.; Wang, P.; Guo, S.; Zhang, X.; Shen, X.; Lu, G.; Su, D.; Huang, X., Ordered $\mathrm{PdCu}$-Based Nanoparticles as Bifunctional Oxygen-Reduction and Ethanol-Oxidation Electrocatalysts. Angew. Chem. Int. 2016, 55, 9030-9035.

(9) Ghosh, A.; Chandran, P.; Ramaprabhu, S., Palladium-Nitrogen Coordinated Cobalt Alloy Towards Hydrogen Oxidation and Oxygen Reduction Reactions with High Catalytic Activity in Renewable Energy Generations of Proton Exchange Membrane Fuel Cell. Appl. Energ. 2017, 208, 37-48.

(10) Liu, H.; Koenigsmann, C.; Adzic, R. R.; Wong, S. S., Probing Ultrathin One-Dimensional Pd-Ni Nanostructures as Oxygen Reduction Reaction Catalysts. ACS Catal. 2014, 4, 2544-2555. 